\title{
ORIGINAL ARTICLE Differential propagation of stroma and cancer stem cells dictates tumorigenesis and multipotency
}

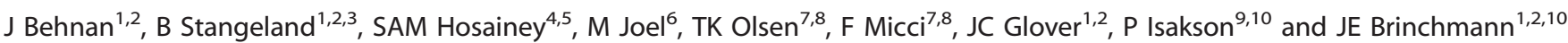

Glioblastoma Multiforme (GBM) is characterized by high cancer cell heterogeneity and the presence of a complex tumor microenvironment. Those factors are a key obstacle for the treatment of this tumor type. To model the disease in mice, the current strategy is to grow GBM cells in serum-free non-adherent condition, which maintains their tumor-initiating potential. However, the so-generated tumors are histologically different from the one of origin. In this work, we performed high-throughput marker expression analysis and investigated the tumorigenicity of GBM cells enriched under different culture conditions. We identified a marker panel that distinguished tumorigenic sphere cultures from non-tumorigenic serum cultures (high CD56, SOX2, SOX9, and low CD105, CD248, aSMA). Contrary to previous work, we found that 'mixed cell cultures' grown in serum conditions are tumorigenic and express cancer stem cell (CSC) markers. As well, $1 \%$ serum plus bFGF and TGF-a preserved the tumorigenicity of sphere cultures and induced epithelial-to-mesenchymal transition gene expression. Furthermore, we identified 12 genes that could replace the 840 genes of The Cancer Genome Atlas (TCGA) used for GBM-subtyping. Our data suggest that the tumorigenicity of GBM cultures depend on cell culture strategies that retain CSCs in culture rather than the presence of serum in the cell culture medium.

Oncogene (2017) 36, 570-584; doi:10.1038/onc.2016.230; published online 27 June 2016

\section{INTRODUCTION}

Glioblastoma multiforme (GBM) is the most common primary tumor of the central nervous system and is highly aggressive, with a median survival of $<15$ months. ${ }^{1}$ The poor prognosis of GBM even after tumor resection followed by radio- and chemotherapy is due to the presence of highly infiltrative cells which escape surgical removal to spread into the normal brain parenchyma, and to the rapid development of a radiation- and chemotherapyresistant cancer stem cell (CSC) population. ${ }^{2}$ Moreover, it is thought that pronounced tumor cell heterogeneity and differentiation plasticity create additional obstacles to treating these lethal tumors. ${ }^{3}$

Tumor formation also involves interactions between the tumor-initiating cells and extrinsic cellular components recruited to the tumor such as fibroblasts, endothelial cells, macrophages and mesenchymal cells or pericytes, all of which contribute to the tumor stroma and vasculature. ${ }^{4-6}$ Although glioma stem cells (GSCs) can give rise to cellular heterogeneity within a glioblastoma tumor through their multilineage differentiation capacity, the existence of non-neoplastic cells within tumor stroma and experiments that trace the origin of such cells in animal models suggest that these cells also play a role in tumor formation. $^{7-10}$

Establishing glioma cell lines with tumor-initiating properties that mimic the parental tumor has been a principal aim in many studies. ${ }^{11,12}$ The sphere culture technique in serum-free medium is widely held to enrich numbers of GSCs in culture. ${ }^{11,13}$
However, this technique has shortcomings such as difficulties in establishing sphere cultures from some patient biopsies, spontaneous differentiation and cell death in some cultures and difficulties in achieving clonal analysis ${ }^{13}$ (Behnan, et al., unpublished observations). On the other hand, adding serum to the primary GBM cell culture medium has been widely accepted to enrich for non-tumorigenic cells and eliminate the tumorigenicity of GSC cultures. ${ }^{11,14}$ One study has reported the tumorigenicity of cells from a primary gliosarcoma at early passage in serum condition, and some commercially available glioma cell lines are tumorigenic in serum condition, but these cell lines were criticized recently. ${ }^{15-17}$ Another widely used method for enrichment of GSCs is cell sorting based on CD133 expression. ${ }^{2,18}$ However, this is not a reliable method as some CD133-negative cells can also be tumorigenic, and some fresh and in vitro-expanded GBM specimens do not express CD133. ${ }^{19,20}$

Thus, the issue of which GBM culture conditions can be used to enrich for tumor-initiating cells is still controversial. To address these questions, we have compared the effects of three different culture conditions, serum-free sphere culture, conventional adherent culture supplemented with $10 \%$ fetal bovine serum (FBS), and adherent culture supplemented with $1 \% \mathrm{FBS}, \mathrm{bFGF}$ and TGF- $a^{21}$ on cells derived from fresh GBM specimens, focusing particularly on their subsequent tumorigenic potential. Furthermore, we demonstrate the mesenchymal properties of a subtype of GBM cells that grow adherently in sphere cultures, and identify

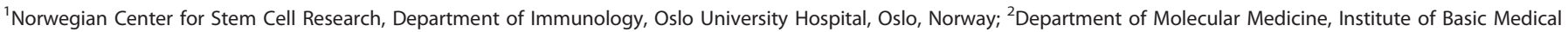

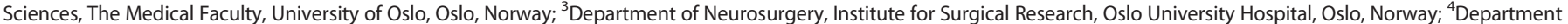

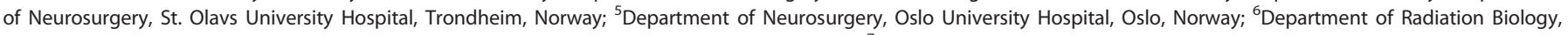

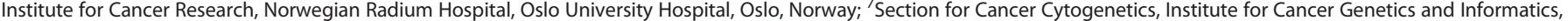

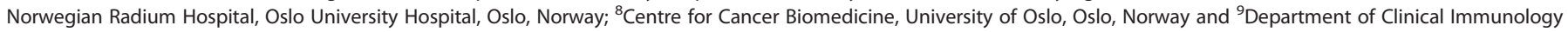

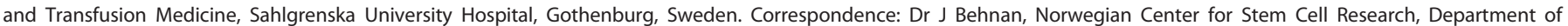
Immunology, Oslo University Hospital, 4950 Nydalen, Oslo 0424, Norway.

E-mail: jinan.bahnan@rr-research.no or jinan_bahnan@hotmail.com

${ }^{10}$ These authors contributed equally to this work.

Received 2 March 2016; revised 15 April 2016; accepted 22 April 2016; published online 27 June 2016
} 
a mini signature of 12 genes that can subtype the GBM tissues in The Cancer Genome Atlas (TCGA) set.

\section{RESULTS}

Characterization of GBM patient samples

In this work we have used ultrasonic aspiration samples of GBM to maximize the heterogeneity of cell types obtained from the tumor. Samples from 21 adult GBM patients were used in this study (see Experimental set-up in Supplementary Table 1). Fresh samples were processed directly or within a few hours after surgery. Pathology analysis on independent core samples confirmed GBM. Characteristics of patients and histological profiles from the pathology analysis are shown in Supplementary Table 2. Preoperative magnetic resonance (MR) images were assessed for tumor locus, infiltration via the corpus callosum, and the tumors were classified according to the study by Lim et al. ${ }^{22}$ (Supplementary Table 2).

Identification of a marker panel that discriminates sphere and serum GBM cultures

To characterize the tumorigenicity of GSCs propagated in vitro under different culture conditions, we utilized three different culture protocols on GBM-derived cells from 21 patients. Two are well-known protocols for GBM-derived cell culture, namely (1) conditions promoting floating sphere formation in serum-free medium (sphere culture) and (2) adherent culture conditions in which medium is supplemented with 10\% FBS (AD10). The third protocol provides adherent culture conditions in which medium is supplemented with $1 \%$ FBS+TGF-a+bFGF (termed AD1), was established by Murrell et al. ${ }^{21}$ for culturing adult human neural stem cells (Supplementary Figure 1a). In both adherent culture protocols, non-adherent cells were removed after $48 \mathrm{~h}$. Under sphere conditions, out of 21 only 14 cultures enriched for cells that actually grew as floating spheres, while the other 7 cultures grew adherently. In our first series of analyses, we focused only on the 14 patient samples that grew as floating spheres. The other seven patient samples were investigated separately (Supplementary Table 1).

Cells within tumor spheres exhibited interpatient morphological differences when dissociated and plated onto an adherent surface. By contrast, cells enriched in AD1 and AD10 serum cultures were predominantly fibroblastoid (Supplementary Figures $1 \mathrm{a}$ and $\mathrm{b}$ ). The In vitro proliferation rate was highest in AD1 cultures (Supplementary Figure 1c). In vitro self-renewal, evaluated by single-cell sorting and limited dilution assay on four samples, was maintained only in sphere cultures, whereas single cells under $A D 1$ and AD10 culture conditions generated only three to seven cells in the first generation and stopped proliferating in the second generation (Supplementary Table 3).

These observations indicate a difference in the cell types that are enriched under the sphere and serum culture conditions. To assess the difference at the protein expression level, we performed flow cytometry analysis on freshly isolated cells and cells expanded under different culture conditions, utilizing a range of 44 surface and intracellular markers previously reported for mesenchymal and neural stem cells (MSCs, NSCs) and GSCs, as well as hematopoietic and endothelial markers (Supplementary Table 4). Of the well-known MSC markers CD73, CD90, CD105, CD9, CD44, CD146, CD166 and PDGFR $\beta$, only CD90, CD44 and PDGFR $\beta$ were expressed in a high proportion of cells in freshly isolated samples and sphere cultures, whereas all abovementioned markers were expressed in a high proportion of cells in AD1 and AD10 (Figure 1a; Supplementary Figure 2; Supplementary Table 4). Specific markers for GSCs (CD15, CD133, CD56, SOX2 and SOX9) were expressed in a low percentage of cells in freshly isolated samples, and increased in sphere condition. Around half of the sphere cultures did not express CD15 and CD133 (three cultures have $<3 \%$ ), while almost all the cultures expressed high levels of CD56, SOX2 and SOX9. GSC markers were essentially absent in AD1 and AD10 cultures. Of pericyte markers, CD248 and aSMA were hardly expressed in fresh samples, in a low or moderate proportion of cells in sphere cultures, but in high proportions of cells in AD1 and AD10. CD105 was high in all serum cultures and very low or undetected in sphere culture (Figure 1b; Supplementary Table 4).

Based on the flow cytometery analysis, we chose eight markers (CD15, CD133, CD56, SOX2, SOX9, CD105, CD248 and aSMA) for immunocytochemical analysis (Figure 1C). The fluorescenceactivated cell sorting (FACS) and immunocytochemical results together indicated that CD56, SOX2 and SOX9 are characteristic of sphere cultures, whereas CD105, CD248, and aSMA are characteristic of serum-supplemented cultures. CD15 and CD133 are specific for sphere culture cells, but do not cover the tumor heterogeneity. Our data suggest that CD56, SOX2, SOX9, CD105, CD248 and aSMA represent the best marker panel that can distinguish cells enriched in sphere and serum cultures.

Cells in sphere cultures have higher neural differentiation potential, whereas cells in serum cultures have higher mesenchymal differentiation potential

To investigate the differentiation potential of GBM-derived cells, we submitted them to neural and mesenchymal differentiation protocols. Under neuronal differentiation conditions, sphere cultures gave rise to cells that expressed a spectrum of neuronal markers (Figure 2a). By contrast, using same laser intensity and confocal settings, in AD1 and AD10 cultures there was some expression of $\beta$-tublll, nestin and Musashi, but little or no expression of MAP2, GFAP, NeuN or DCX (Figure 2a). Under mesenchymal differentiation conditions, osteogenesis was high in both AD1 and AD10 and low in sphere cultures (Figure 2b), whereas chondrogenesis shown by aggrecan staining was strong in AD10, weak in AD1 and absent in sphere cultures (Figure 2b). None of the culture conditions supported adipogenesis (Figure 2b). Lacking the differentiation potential into adipocytes was recently explained by the expression of Gremlin 1 (GREM1) in a subset of bone marrow-MSCs (BM-MSCs) named osteochondroreticular. ${ }^{23}$ We checked the expression of GREM1 in our microarray data set, and noticed high-expression levels for this gene in both AD1 and AD10 compared with Sp cultures (Supplementary Figure 3). Thus, cells in sphere and adherent cultures were characterized predominantly by multilineage neural and bilineage mesenchymal differentiation capacities, respectively.

Global mRNA profiling and CGH show similarity between sphere cultures and original tumor, and between serum cultures and MSCs

To evaluate these differences at the transcriptional level, we carried out global mRNA expression analysis using microarrays on cells in different culture conditions. Principal component analysis demonstrated a clear separation of AD1 and AD10 from sphere cultures and freshly obtained tumor cells (Figure 3a). Hierarchical cluster showed that the sphere cultures were more closely related to the fresh tumor cells, whereas AD1 and AD10 clustered together with other MSC-like cells from brain, BM and adipose tissue (Figure $3 \mathrm{~b}$ ). We also performed hierarchical clustering using the genes coding for our marker panel of GSC versus MSC-like cells. The resulting dendrogram gave similar clustering to the global analysis (Figure 3c). This, together with box plots of mRNA relative transcript expression (Supplementary Figure 4), indicates that the marker panel clearly distinguishes cells in sphere condition from those in serum conditions, also at the transcriptional level. 
To test whether the cultured cells had genomic aberrations related to those in freshly isolated GBM samples, we carried out a comparative genomic hybridization (CGH) analysis, using cells at low passages (P4-P7). Cells enriched in sphere cultures shared some genomic aberrations with the original tumor. Cells enriched in AD10 cultures exhibited only a few genomic aberrations. By
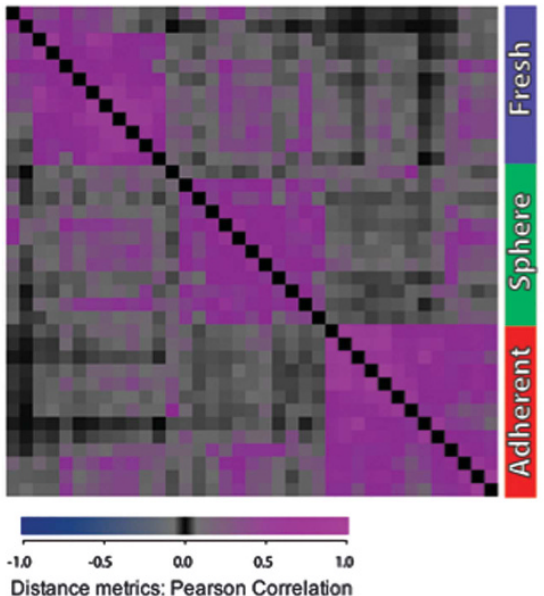
Linkage: WPGMA

b
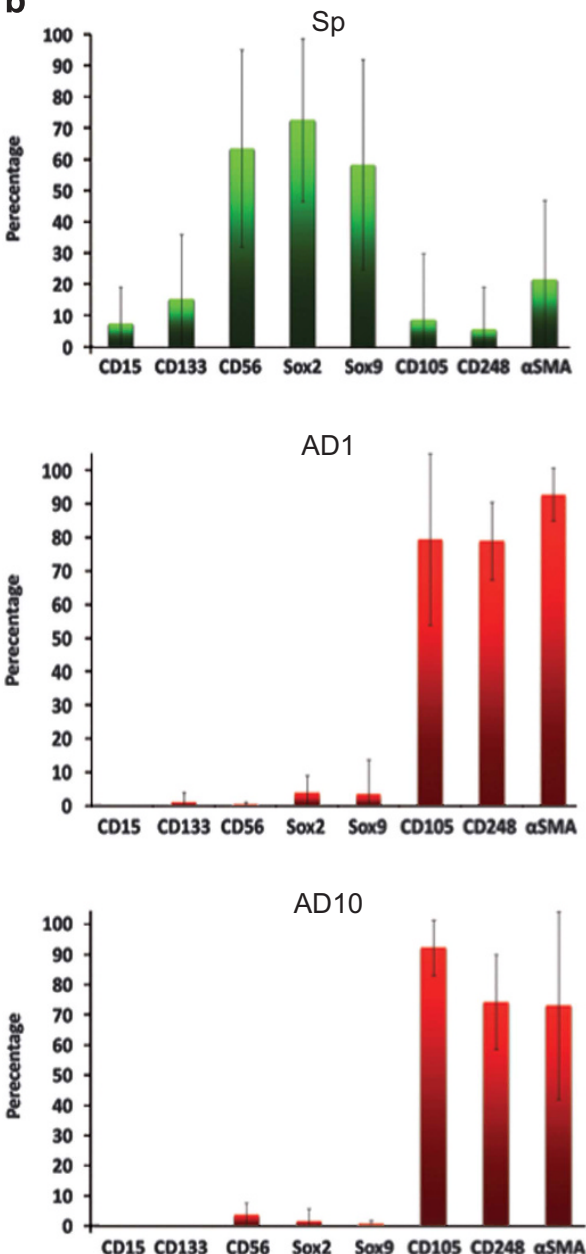

C
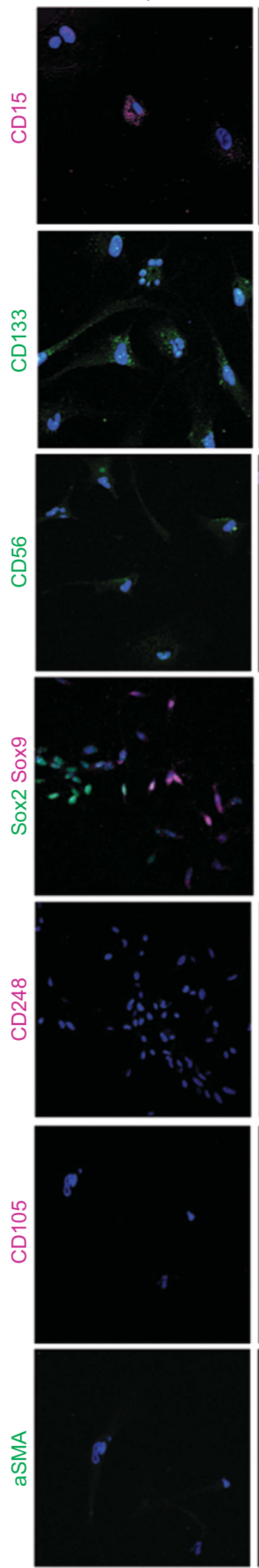

AD1
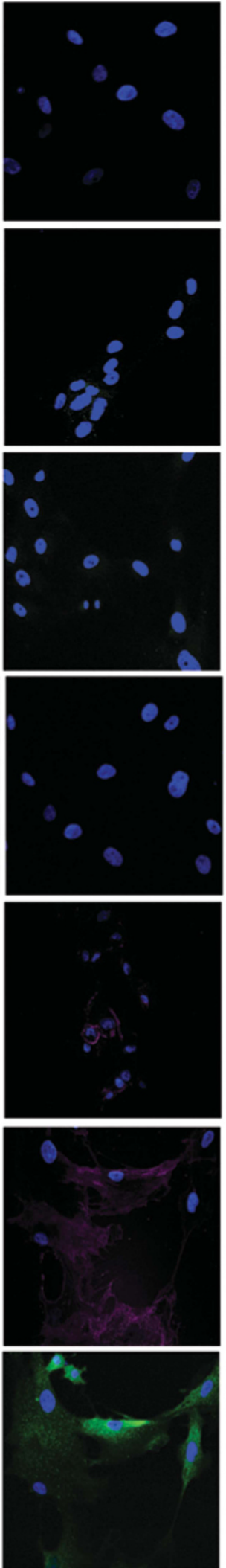

AD10
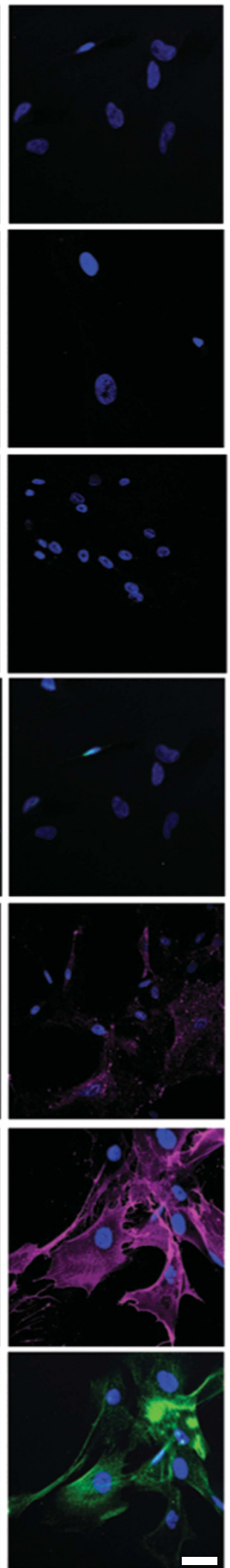
contrast, cells enriched in AD1 cultures displayed balanced genomes by $\mathrm{CGH}$, which means that they are normal stroma cells, not tumor cells (Supplementary Table 5).

MSC-like cells have perivascular localization, while GSCs are distributed within tumor stroma in fresh GBM tissues

To confirm that the panel of markers that distinguishes sphere from serum cultures is expressed by cells in the original GBM tissue, we performed immunohistochemistry (Figure 4). We found expression of GSC-associated markers CD15, CD133, CD56, SOX2 and SOX9 in cells distributed within the tumor stroma, but limited or absent expression in pericytes surrounding the blood vessels. In contrast, CD105, CD248 and aSMA, as well as other mesenchymal markers were prominent in perivascular cells, although some expression of CD248 was present outside the perivascular niche. Neural markers (GFAP, $\beta$ tublll, MAP2, nestin) were highly expressed throughout the tumor, often close to the vessels (Figure 4). Thus, cells preferentially expanded in sphere- versus serum-containing cultures were differentially localized in primary GBM tissues, and the differentially expressed markers were maintained during in vitro culture.

Freshly isolated GBM cells and sphere cultures are tumorigenic To examine the tumorigenic potential of cells enriched in the different culture conditions, we transplanted these cells into one cerebral hemisphere of immunocompromised mice (Supplementary Table 6). Mice injected with cells from sphere cultures developed neurological symptoms more quickly than mice injected with cells from the corresponding fresh tissue samples ( $P<0.01$, log-rank test; Figure 5a). Although many factors contribute to the rapidity of tumor formation and the corresponding onset of neurological symptoms, since the number of injected cells were equal this will in most cases mean that cells from sphere cultures developed tumors more rapidly than cells from fresh tumor samples. Both types of $\mathrm{Fr}$ and $\mathrm{Sp}$ cells formed highly invasive tumors that spread into the opposite hemisphere (Figures $5 \mathrm{~b}$ and $\mathrm{c}$ ). The only exception was the sphere culture derived from patient sample T1311, a secondary GBM, which induced a low grade-like tumor and animals were killed without neurological symptoms (Supplementary Figure 5a). The survival times of mice injected with cells from sphere cultures varied substantially (Supplementary Table 6). Cells from sphere cultures also appeared to produce less angiogenic tumors than cells from the matched freshly isolated samples, based on the expression of CD31 and the pericyte and mesenchymal markers included in our panel (CD248, CD105 and aSMA) (Figure 5d; Supplementary Figures $5 b-d)$. By contrast, cells from both types of serum cultures generated no tumors at all (Figure 5a; Supplementary Figure 6). Thus, sphere culture conditions preserved the tumorigenicity of the freshly isolated tumor cells, whereas serum culture conditions depleted of non-adherent cells after $48 \mathrm{~h}$ were non-tumorigenic.

Mixed cell cultures: serum culture conditions can support the growth of a tumorigenic cell subpopulation

It is controversial whether tumor-initiating cells can be maintained in serum-supplemented culture. Some studies even report that serum can be used as a differentiation factor that abolishes tumorigenicity completely within 7 days. ${ }^{14}$ In the experiments described above, the creation of adherent serum-supplemented cultures included the removal of non-adherent cells after $48 \mathrm{~h}$, as is common practice for primary cultures of adherent cells. Different properties resulted when we did not remove the nonadherent cells. This was done in 4 of the patient samples using AD10 serum conditions and in 2 of the samples using AD1 serum conditions. After 4-7 days, we found that clusters of predominantly bipolar cells had formed on top of the adherent fibroblastoid cells, creating what we call a 'mixed cell culture' (Figure 6a). These cultures continued to proliferate (Figure $6 \mathrm{~b}$ ). FACS analysis using our previously established panel of markers showed that mixed cell cultures had an expression profile that was a hybrid between sphere and serum cultures (Figures $6 \mathrm{c}$ and $\mathrm{d}$; Supplementary Table 7). Also, CGH analysis showed some of the same genomic imbalances as in the fresh samples (Supplementary Table 5). On intracerebral transplantation, cells from mixed cell cultures generated lethal tumors, in contrast to cells from the AD1 and AD10, although survival was longer than tumors derived from the sphere cultures from the same patient $(P<0.01$, log-rank test) (Figure 6e). The tumors were invasive, spreading to the opposite hemisphere, and appeared to be more angiogenic than those derived from sphere cultures (Figures $6 f$ and g; Supplementary Figure. 7).

Mixed cell cultures transferred to sphere culture conditions at P4-P8-formed spheres or semi-attached spheres and continued to proliferate, whereas serum cultures transferred to sphere culture conditions stopped proliferating and died off within 3 months. Thus, it was not serum supplementation per se, but rather the early removal of non-adherent cells from plated fresh tumor cells that abolished tumorigenicity in cells from serum cultures.

Transfer of sphere cultures to AD1 serum culture conditions does not abolish tumorigenicity

To test the effect of serum supplementation on the tumorigenicity of sphere cultures, we transferred sphere cultures into AD1 conditions. The cells remained proliferative in the new medium (Figure 7a), and FACS analysis showed decreased expression of CD133 and CD15, while there was a trend for increase in SOX2 and SOX9 $(P=0.068)$ (Supplementary Table 8$)$. Intracranial transplantation of these cells from two of the patients after P6 and P8 induced tumors more quickly than did the original sphere cultures,

Figure 1. Identification of a marker panel that distinguishes sphere cultures from serum cultures. (a) Quantified FACS data were used for hierarchical clustering with a distance matrix to determine the level of co-expression. Expression of 44 known markers for GSCs, MSCs, NSCs and other stroma-contributed lineages was assessed in 37 cell cultures. Each square in the chart represents the Pearson's correlation between the expression levels of all 44 tested markers in 2 cell cultures (purple representing the highest and dark blue the lowest correlation, full size figure with complete sample names and marker designation can be seen in Supplementary Figure S2b). This analysis showed separation into three categories: fresh, spheres and the adherent cultures. The last category encompassed cells grown in AD1 and AD10. Additional analysis is presented in Supplementary Figure S2. (b) Hybrid FACS expression profile of selected 8 markers in 13 patients in sphere conditions (Sp), 7 in AD1 serum cultures and 6 in AD10. The hybrid profile shows that CD133 and CD15 are moderately and differentially expressed among patients in Sp condition, while are almost absent in both AD1 and AD10. CD56, SOX2 and SOX9 are consistently high in sphere and very low in both AD1 and AD10. CD105, CD248 and $\alpha$ SMA are low or moderately expressed in sphere and highly expressed in AD1 and AD10. (c) Immunofluorescent staining of a selected marker panel (CD15, CD133, CD56, SOX2, SOX9, CD248, CD105, $\alpha$ SMA) showed that the first five markers are expressed in sphere culture (Sp) represented in the first column, and absent in AD1 and AD10 shown in the second and third columns, while CD248, CD105 and $\alpha$ SMA were highly expressed in serum cultures and absent in Sp. Same laser intensity and confocal settings were used for comparison. Scale bar, $20 \mu \mathrm{m}$. 


\section{a}

DAPI TubllI MAP2
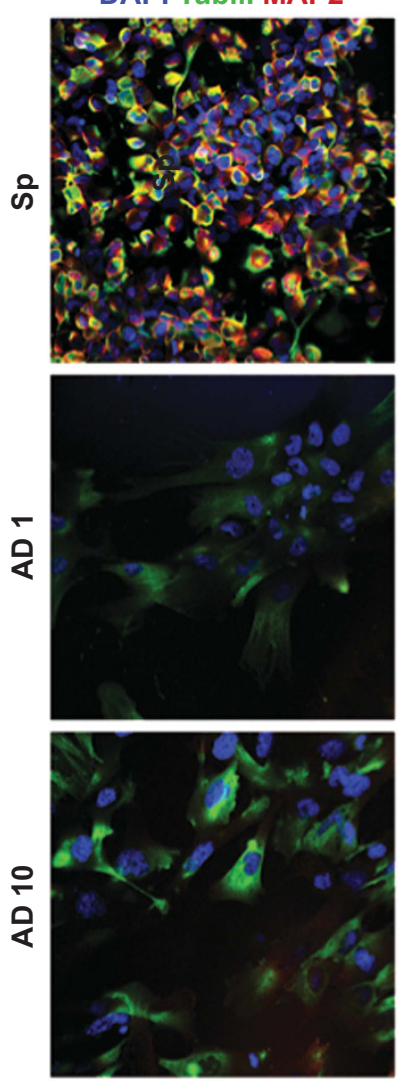

b
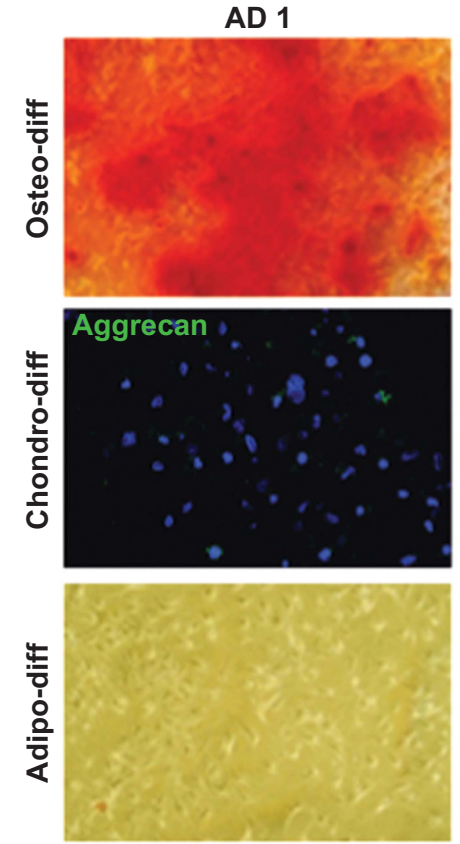

DAPI GFAP Nes
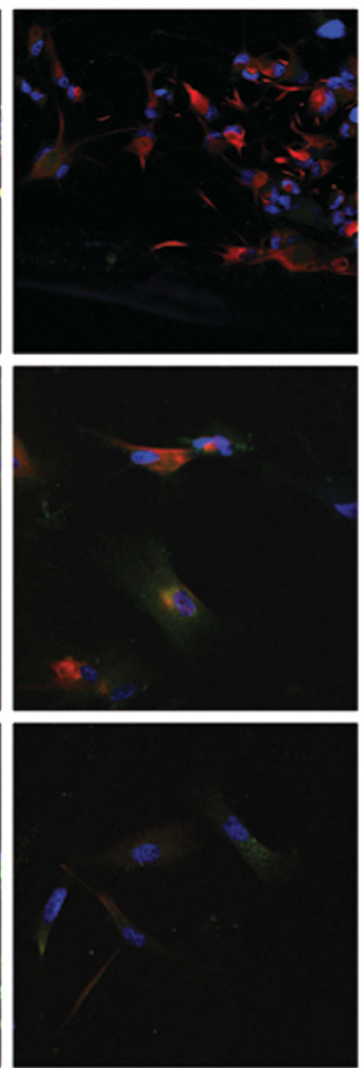

AD 10
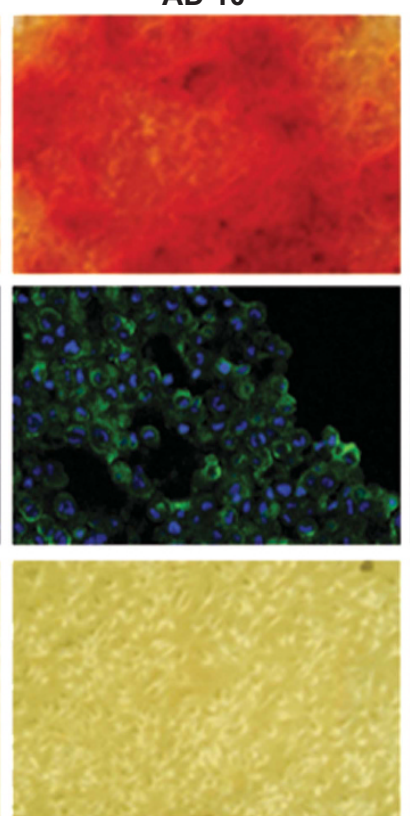

DAPI NeuN

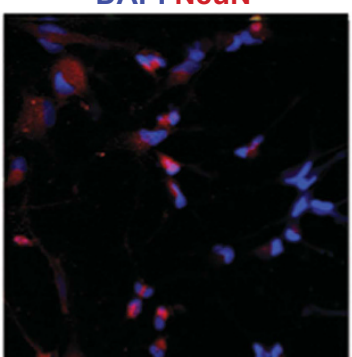

DAPI DCX Musashi
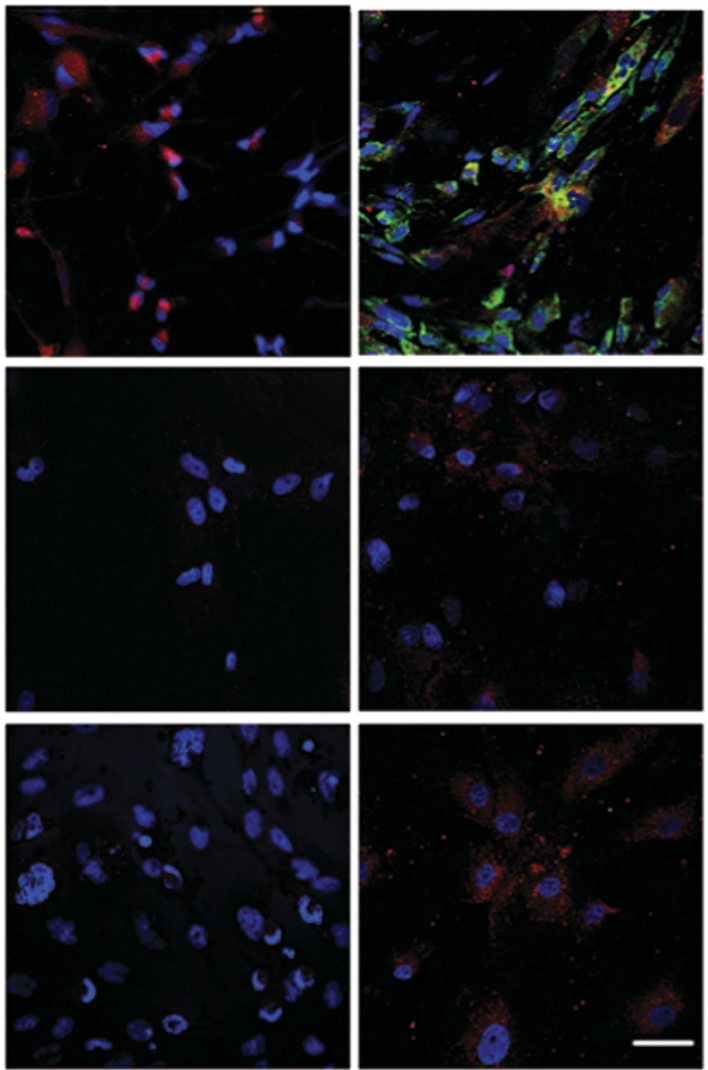

$\mathrm{Sp}$
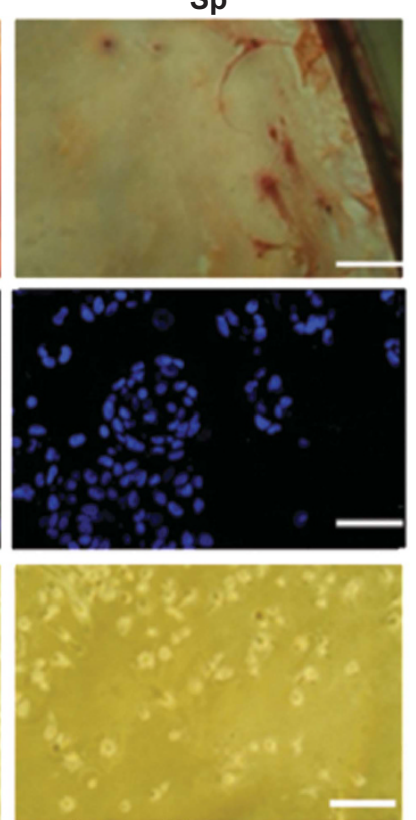

Figure 2. Sphere culture-enriched cells showed neural differentiation potential, whereas serum culture-enriched cells showed some mesenchymal properties. (a) Immunofluorescent staining of neural lineage markers ( $\beta$ Tublll, MAP2, GFAP, NeuN, DCX and Musashi) after 3 weeks of in vitro differentiation using the same laser intensity and confocal settings. Sphere culture (Sp) expressed high levels of the neural differentiation panel, while serum cultures, AD1 and AD10, expressed only BIII and Musashi at lower level. Scale bar, $20 \mu \mathrm{M}$. (b) First row: osteogenic differentiation potential, after 2 weeks, showed that serum cultures, AD1 and AD10, have strong osteogenic differentiation as opposed to Sp culture. The staining was visualized with alizarin red staining for calcium deposits. Scale bar, $250 \mu \mathrm{m}$. Second row: aggrecan staining showed that chondrogenic differentiation was strong in AD10, weak in AD1 and absent in Sp culture. Scale bar, $50 \mu \mathrm{M}$. Third row: oil red staining for lipid droplets showed that none of the culture condition-derived cultures could differentiate into adipocytes. Scale bar, $250 \mu \mathrm{M}$. 
a
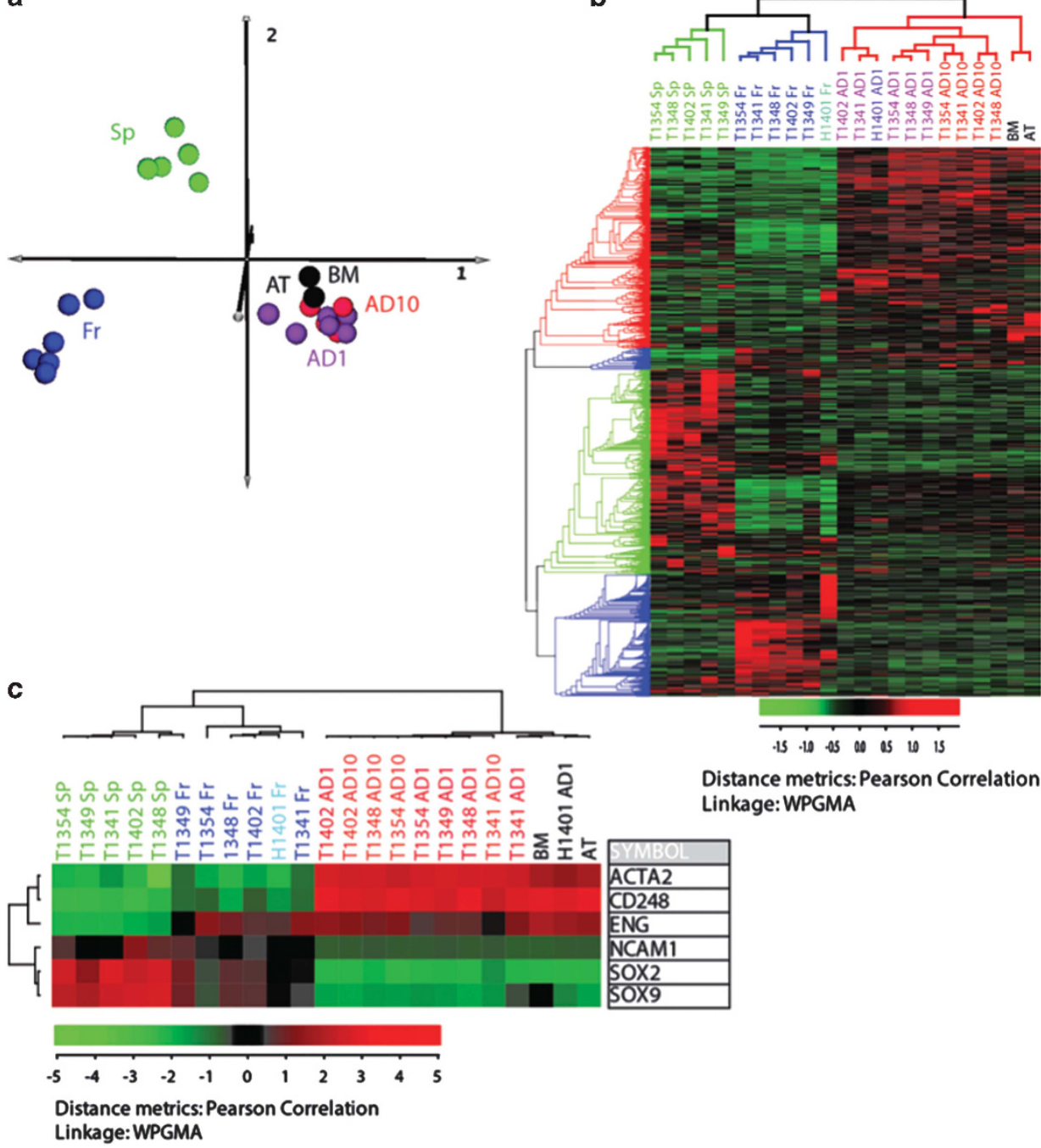

Distance metrics: Pearson Correlation Linkage:WPGMA

b
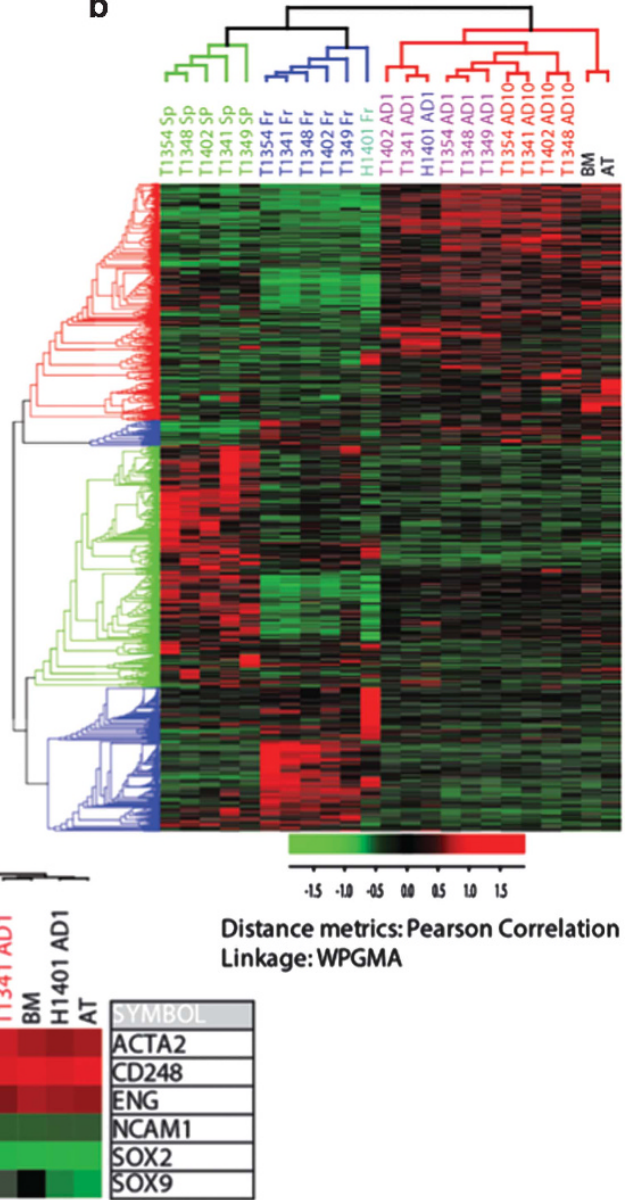

SOX2

Figure 3. Global mRNA profiling confirmed that sphere cultures are more related to their original tumors, whereas serum cultures are more related to MSCs. (a) Global expressional analysis showed clear separation between fresh tumors (Fr) and cultured cells in sphere (Sp) or serum conditions-AD1 and AD10. To visualize similarities or dissimilarities of our data sets, we used principal component analysis (PCA) of gene expression. PCA simplified the visualization of individual and group differences: Sp cultures were clearly separated from the parental tumors (Fr), while AD1 and AD10 cultures (purple and red spheres, respectively) were closer to BM- and AT-MSC control lines (black), respectively. (b) Unsupervised hierarchical clustering using 8700 genes with the most variable expression. Low expression is shown with green color while high expression is shown in red. Dendogram colors specify spheres, Sp (green) and fresh tissues, Fr (blue) while purple and red represent AD1 and AD10, respectively. AD1 and AD10 clustered together with BM- and AT-MSC. Fresh samples and sphere cultures clustered together with the main branch, which contains all sphere cultures together and another branch for parental fresh tumors. One human brain tissue sample from an epileptic patient clustered together with the Fr group. The expression values were log2 transformed. (c) An hierarchical clustering chart showing the expression of the seven-gene panel that can classify cell cultures into two groups: (a) Spheres and tissues (blue) that clustered together and (b) adherent cultures (red) that clustered with MSCs controls. The markers of Sp (CD133/PROM1, CD56/NCAM1, SOX2/SOX2, SOX9/SOX9) have high-expression levels in Sp cultures, moderate or low in Fr, and low in serum cultures and MSCs. CD248/CD248, CD105/ENG and $\alpha$ SMA/ACTA2 are highly expressed in serum cultures and MSC controls, moderate or low in Fr samples and Sp cultures. The expression values were log2 transformed.

$(P<0.003$, log-rank test), while the third patient sample transplanted after P3 did not have significant survival difference from its original sphere (Figure $7 \mathrm{~b}$ ), and the tumors seemed to be more angiogenic (Figures 7c and d; Supplementary Figures 8a-c).

Exposing cells to serum has been shown to induce epithelialto-mesenchymal transition (EMT), which plays a role in angiogenesis. $^{24}$ Moving cells from sphere condition to AD1 showed an increase in the mRNA expression of EMT-related genes (Figure 7e). Thus, AD1 condition did not abolish the tumorigenicity of sphere cultures, but rather provided cells which induced rapid and more angiogenic tumors.
Patient samples that grow adherently under sphere culture conditions exhibit mesenchymal properties and features of mixed cell cultures

Among the 21 primary cultures that we established in sphere condition, seven cultures nevertheless grew adherently, suggesting a difference in adherence that precluded the formation of spheres. These 'adherent' sphere cultures had a high proliferation rate (Figure 8a), and exhibited osteogenic and chondrogenic (Figure 8b), but not adipogenic differentiation potential (not shown). Because these patient samples have mesenchymal properties, we will hereafter refer to them as the mesenchymal 

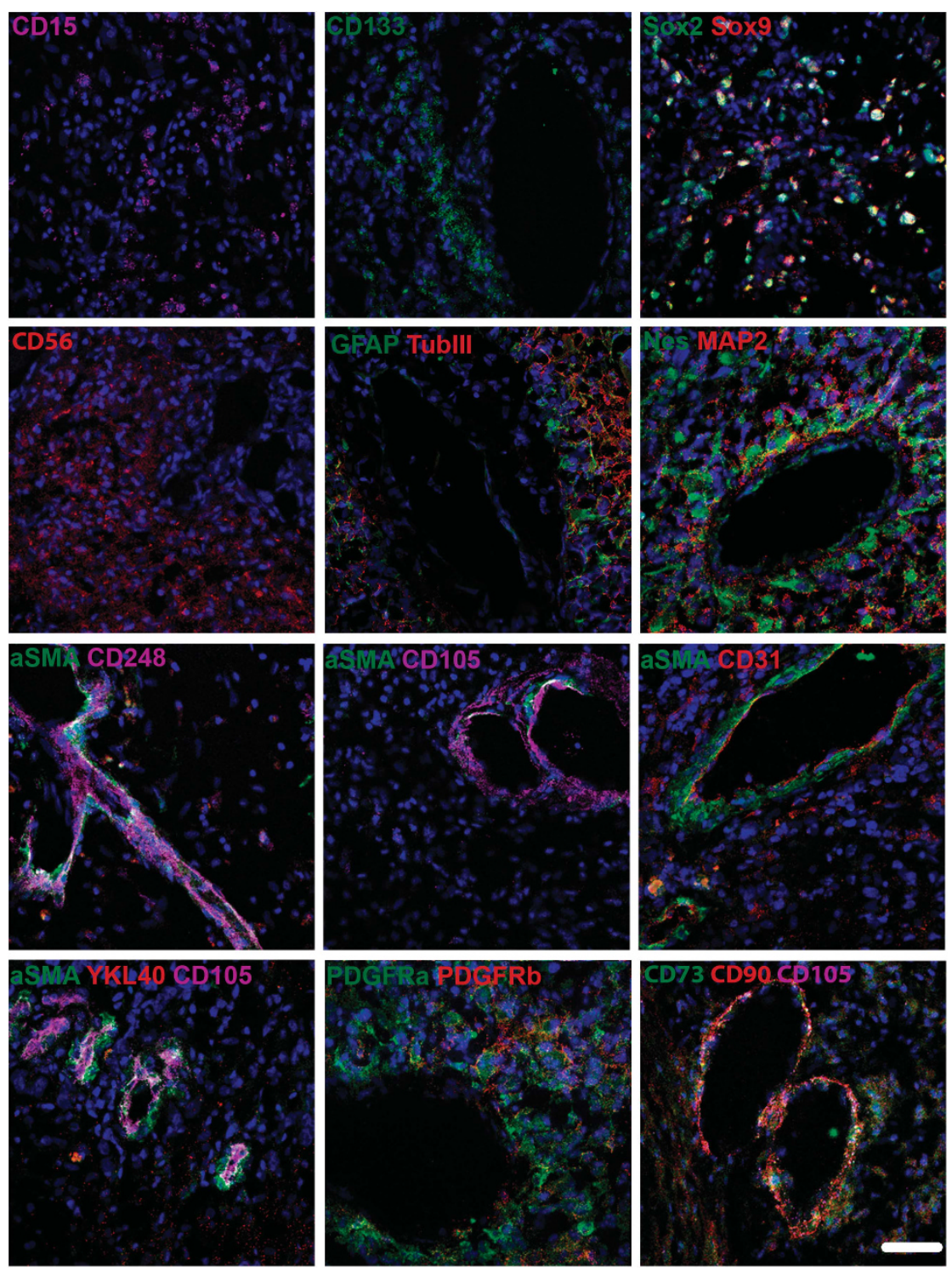

Figure 4. Cells expressing MSC markers have perivascular localization, while cells expressing GSC markers spread within tumor parenchyma in fresh human GBM tissue. Immunofluorescent staining for a selected panel of GSC markers (CD15 (red), CD133 (green), SOX2 (green), SOX9 (red), CD56 (red)), neuronal markers (GFAP(green), $\beta$-Tublll(red), Nestin (green) and MAP2 (red)), pericyte and endothelial markers ( $\alpha$ SMA (green), CD248 (purple), CD105 (purple) and CD31 (red)), GSC and MSCs Marker (YKL-40 (red), PDGFR- $\alpha$ (green), PDGFR- $\beta$ (red), CD73 (green), CD90 (red). The GSC and neuronal markers have limited or undetected expression in perivascular cells. Pericyte markers ( $\alpha$ SMA, CD248, and CD105) have strong expression specifically in perivascular cells except CD248 which could stain a few cells spreading away from the vasculature spot. The other MSC markers were expressed in both perivascular and tumor parenchyma. Scale bar, $20 \mu \mathrm{m}$.

group ('Mes'), and sphere culture derived from these patients as 'Sp-Mes'. Sp-Mes cells from two different patient samples were transplanted intracerebrally into severe-combined immunodeficiency (SCID) mice and generated invasive tumors that spread to the opposite hemisphere (Figure 8c). Interestingly, FACS analysis showed a hybrid expression profile similar to that of the mixed cell cultures (Supplementary Table 9; Supplementary Figure 9).

Comparing the mesenchymal and the sphere-forming patient subgroups in terms of the expression of EMT-related genes showed that the mesenchymal group of both freshly isolated cells (Fr-Mes) and sphere cultures (Sp-Mes) had higher EMT expression levels than $\mathrm{Sp}$ and their parental fresh tumor ( $\mathrm{Fr}$ ) (Figure 8d).

The hybrid expression profile of the six-marker panel, combined with mesenchymal differentiation capacity and EMT gene profile, prompted us to make a more detailed molecular characterization of the Mes-subgroup sphere cultures. To accomplish this, we used the established classification gene set of Bhat et $a l^{25}$ to classify sphere cultures into two distinct groups: mesenchymal and proneural. Hierarchical clustering using the 1000 genes set of Bhat et al. $^{25}$ showed that Sp-Mes expressed predominantly the mesenchymal signature of Bhat et al. ${ }^{25}$ (Supplementary Figure 10), while four of the five sphere cultures clustered together and expressed predominantly the proneural signature of Bhat et al's cluster $2 .{ }^{25}$ Comparison of gene expression profiles of $\mathrm{Sp}$ with proneural signature and $\mathrm{Sp}$-Mes with mesenchymal signature identified 118 differentially expressed genes (DEGs) (Figure 8e). The four clustered sphere cultures expressed predominantly classical and proneural genes, whereas the Sp-Mes cultures expressed predominantly mesenchymal genes (Supplementary Figures 11a and b). Previously, TCGA has classified all GBM tissues into four distinct transcriptional subtypes: Proneural, 


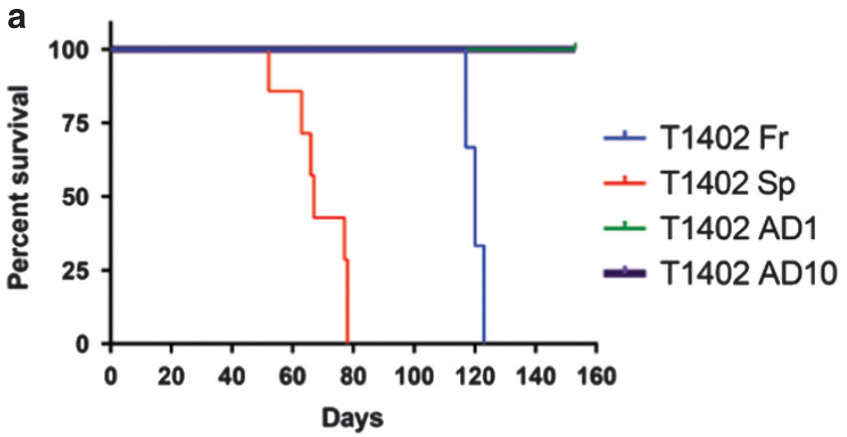

b
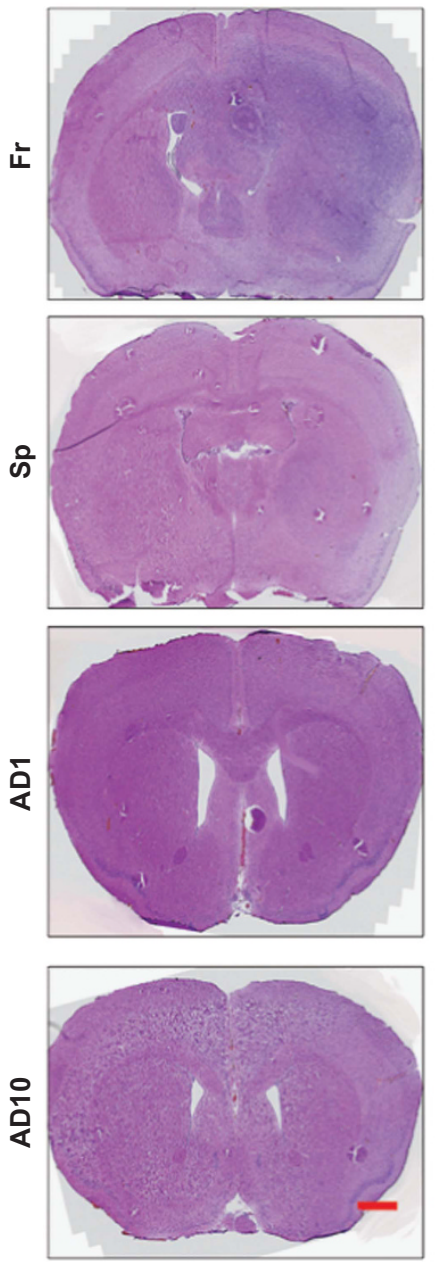

c
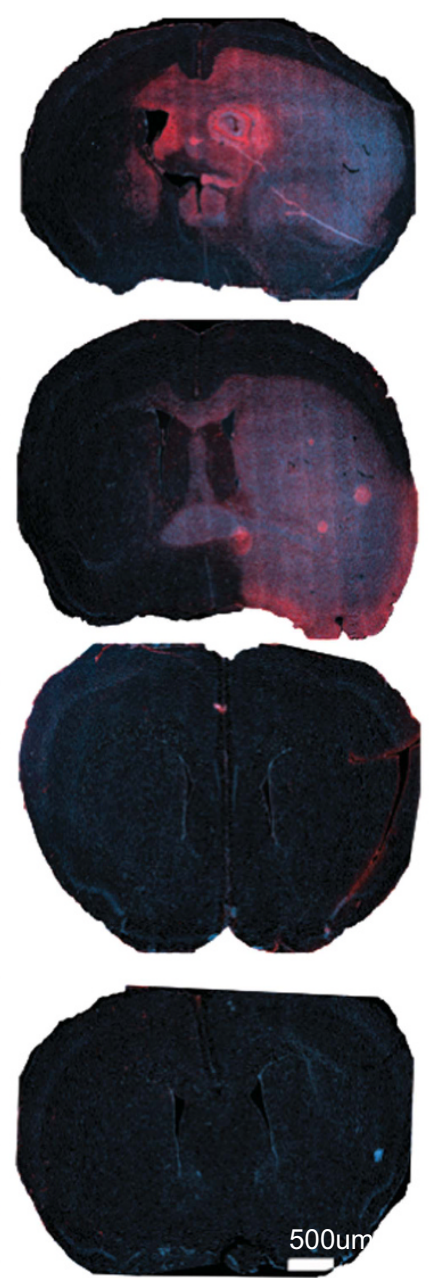

d
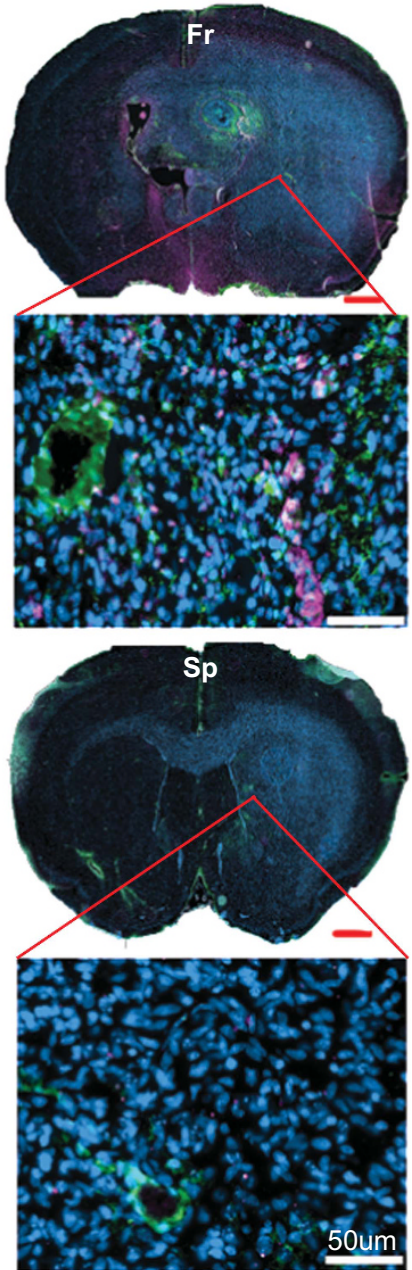

Figure 5. Tumorigenecity is conserved in fresh and sphere cultures, but not in serum cultures. (a) Kaplan-Meier survival analysis of fresh tumor (Fr) and cultured cells under different conditions showing that only Fr and Sp culture induced tumor, but not serum cultures AD1 and AD10. The Sp-induced tumor has significantly shorter time than the Fr-induced one $(P<0.01)$. (b) Representative H\&E staining of tumors induced by $\mathrm{Fr}, \mathrm{Sp}, \mathrm{AD} 1$ and AD10. Scale bar, $1000 \mu \mathrm{M}$. (c) Immunofluorescent staining with human-specific nestin (red) and DAPI showing the infiltrative malignant glioma feature in both Fr and Sp. Scale bar, $500 \mu \mathrm{m}$. (d) An overview and zoom-in image of immunofluorescent staining of CD31 (green), $\alpha$ SMA (red) and DAPI. Higher expression for CD31 and $\alpha$ SMA was noticed in Fr compared with Sp. Scale bar, $500 \mu \mathrm{m}$ in overview image and $50 \mu \mathrm{m}$ in zoom-in.

Mesenchymal, Classical And Neural using 840-gene set. ${ }^{26}$ By comparing the list of 118 DEGs with TCGA 840-gene signature, we identified 12 genes that categorized the TCGA-GBM tissues into three subtypes: Mesenchymal, Classical and Proneural (Figure 8f). While our subtype using the 12-gene signature overlapped with the TCGA subtype of Mesenchymal, Classical and Proneural categories, this 12-gene signature could not clearly define the Neural subtype. The neural tumors from TCGA were spread between our Proneural and Classical subtype mainly (Supplementary Figure 11b). Merging all our 36 expression profiles, including the BM-MSCs and adipose tissue stem cells (AT-MSC), with the 173 GBM tissues of the TCGA core set showed that the Mesenchymal subtype is divided into two groups: (1) 'Tissue mesenchymal' identified with three genes (TGFB1, S100A4 
and DAB2) that were predominantly expressed in GBM tissues (approximately one third of the Mesenchymal subtype), (2) 'Culture mesenchymal' identified with three genes (COLA1A1, COLA1A2 and THBS1) that were highly expressed in cells of AD1,
AD10 cultures and group of GBM tissue (two thirds of the mesenchymal subtype) (Supplementary Figures 11a and b).

Interestingly, the subtyping of the parental tumors of both $\mathrm{Sp}$ and Sp-Mes was different from these sphere cultures, where three a

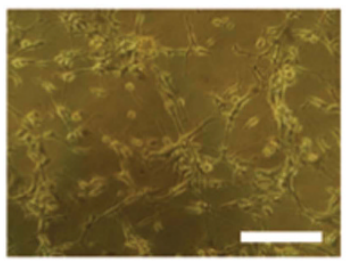

C
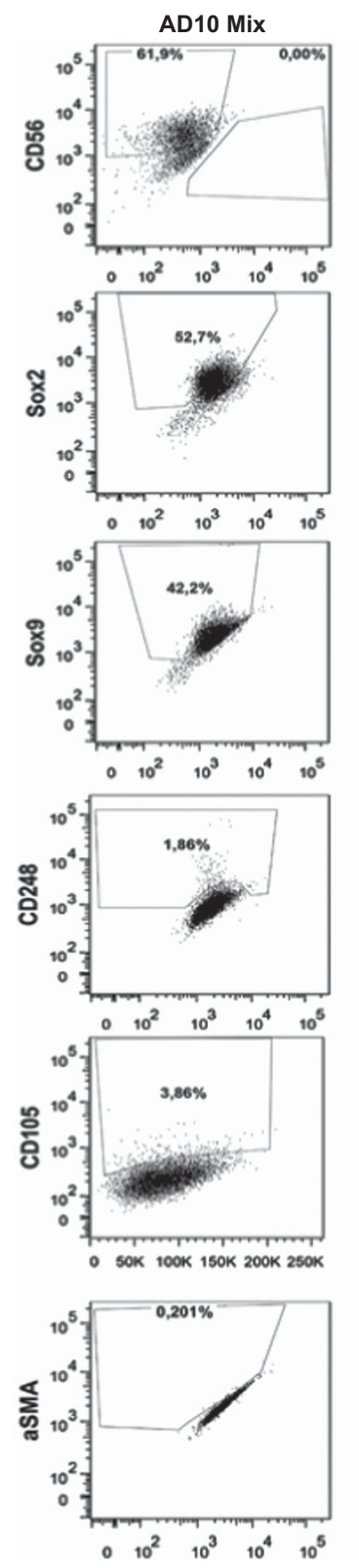

b
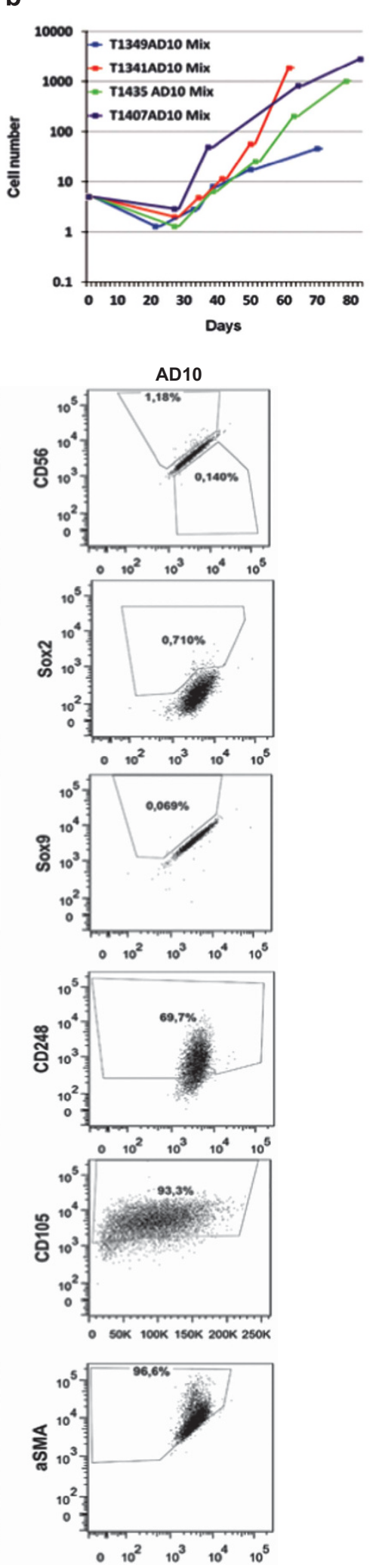

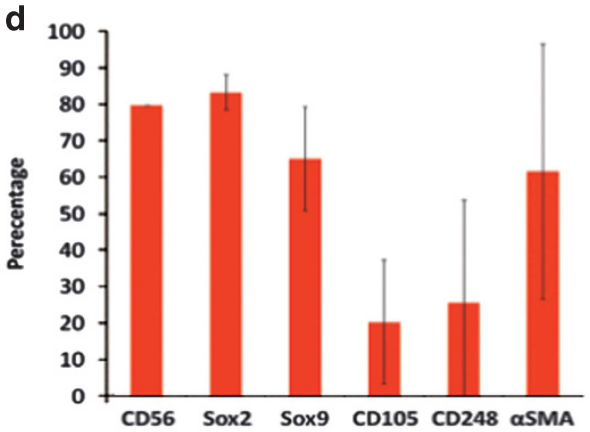

e

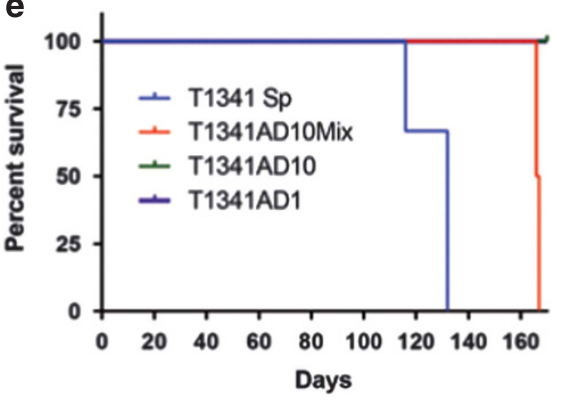

f

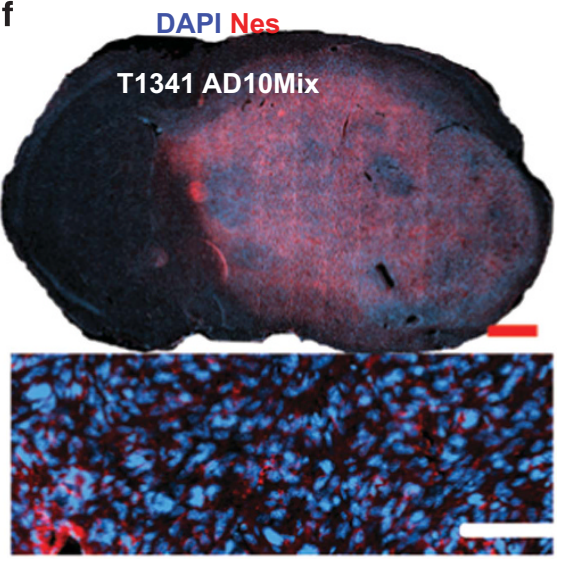

$\mathbf{g}$

DAPI CD248 CD31 aSMA

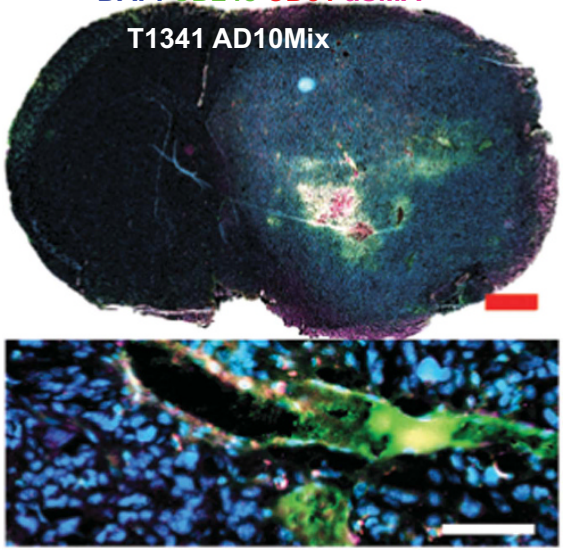


of the parental tumors of Sp-Mes (Fr-Mes) were of Proneural subtype, while the parental tumors of all Sp were of Mesenchymal subtype (Supplementary Figure 12).

Thus, the Sp-Mes cultures exhibit several biological properties of MSCs, and using the subtyping by Bhat et $a l^{\prime 25}$ demonstrates that the Sp-Mes cultures have a mesenchymal expression profile. Further analysis revealed a differentially expressed set of 12 genes shared between our 118 genes and the 840 genes of TCGA, which alone could subtype the TCGA-GBM tissues into 3 main subtypes.

\section{DISCUSSION}

Identifying optimal culture conditions for GBM has been the aim of many studies. ${ }^{11-13}$ Although sphere culture is considered the gold standard for propagating GBM in vitro, it has many limitations, including spontaneous differentiation and cell death in some cultures, difficulties in achieving clonal analysis, and most importantly, the success rate in establishing primary sphere cultures ranges only from 50 to $70 \%^{11,27}$ (Behnan et al., unpublished). This means that at least one third of the patients will be missed in studies using sphere culture to characterize and target GSCs. However, including samples that grow adherently under sphere culture conditions will allow growth of tumorigenic cells from a wider range of patients. To cover the heterogeneity among GBM patients, we have suggested a panel of markers (high CD56, SOX2, SOX9, and low CD105, CD248, aSMA) that can distinguish tumorigenic cultures from nontumorigenic cultures derived from different patients. For the first time, we have introduced the concept of the mixed cell culture, tumorigenic cells grown in serum conditions, and characterized it phenotypically and functionally. Finally, we characterize the group of sphere cultures that grow adherently (Sp-Mes) and identify 118 genes differentially expressed between our sphere and $\mathrm{Sp}$-Mes cultures. Twelve genes from this set were shared with the 840 genes of TCGA. ${ }^{26}$ Interestingly, these 12 genes alone were able to subtype the GBM tissues from TCGA into three subtypes almost as accurately as the original signature of 840 genes.

\section{MSC-like cells in serum conditions}

We consider the type of cells that are derived from GBM samples grown under serum culture conditions to be MSC-like cells and not MSCs. Although our transcriptome analysis and the protein expression of a range of MSC markers indicate similarity to MSCs, the typical multilineage differentiation capacity was limited. These cells showed differentiation potential into osteoblasts and chondrocytes. However, the chondrogenic differentiation of adherent cultures that we carried out in an alginate scaffold was different from typical chondrogenic differentiation of BM- and ATMSCs in this system, where our cells did express the cartilagespecific proteoglycan, aggrecan, but not type II collagen, which is another typical differentiation marker for chondrocytes derived from BM-MSCs. ${ }^{28}$ Moreover, none of the serum cultures exhibited adipogenic differentiation capacity. This, in addition to the highexpression level of GREM1 in AD1 and AD10 cells suggests a similarity between mesenchymal stromal cells in GBM and osteochondroreticular stem cells.

\section{Identification of a marker panel for tumorigenic GBM cell cultures}

Currently, there is no universal marker for GSCs. To look for markers that distinguish sphere culture cells from adherent serum culture cells, we performed extensive phenotype analysis of surface and intracellular markers of GSCs, MSCs and NSCs. The marker panel that we picked from our FACS analysis contains three markers (CD56, SOX2, and SOX9) that were previously reported for GSCs and/or NSCs. CD56 is seldom used as a GSC marker, ${ }^{29}$ but it has high to moderate expression in 19 out of our 20 sphere cultures. SOX2 has been shown to be expressed in a subset of GSCs that belong to Classical, Neural and Proneural subtypes but not the Mesenchymal subtype. ${ }^{30}$ Our FACS analysis showed that SOX2 was expressed in all 20 sphere cultures, but it was relatively low in one secondary GBM which induced a low grade-like tumor in vivo (T1311 Sp). The difference in SOX2 expression within the mesenchymal subtype between this work and our work could be due to the methods used. They have used immunofluorescent staining, while we used a quantification method with FACS. Actually, in their study we noticed perinuclear staining for SOX2 in the Mesenchymal subtype cultures, but these cultures were defined as SOX2 negative. ${ }^{30}$ SOX9 expression has recently been reported in GBM tissue and the U251 glioma cell line, but not in primary cultures. ${ }^{31}$ In our set, SOX9 has high to moderate expression in 17 sphere cultures, absent in 1 secondary GBM (T1311 Sp), and $<2 \%$ in 2 other sphere cultures. The other marker of MSCs and pericytes in our panel CD105 has been previously reported to stain for endothelial markers in GBM tissue, ${ }^{32}$ while CD248 and aSMA were reported to be expressed by pericytes in GBM tissue and GSC-differentiated pericytes. ${ }^{8}$ Our data also show colocalization of CD248 and aSMA in pericytes of GBM tissue. Although CD133 was not expressed in around half of our sphere cultures, this marker is one of the most frequently used CSC markers, where tumorigenicity was associated with the CD133 positive fraction only. 2,18 Importantly, many disadvantages have been reported with using this marker: (1) mRNA expression is not correlated to protein expression, ${ }^{33}$ (2) CD133 expression varies during cell cycle, $^{34}$ (3) the CD133negative population has tumor-initiating capacity ${ }^{20}$ and (4) CD133 is not expressed in all patient samples. In our data set, 11 out of 20 of primary sphere cultures expressed CD133 ranging from 2 to $48 \%$. However, we show that CD133-negative cells have in vitro self-renewal, serial clone formation and tumorigenic capability. Also, CD133 expression is affected by changing culture conditions.

CD15 has also been said to enrich for GSCs. ${ }^{35}$ However, only 6 out of our 20 primary sphere cultures expressed CD15, ranging from 1 to $34 \%$ of the total cell population.

Figure 6. Adherent serum cultures that express GSC markers 'mixed cell culture' are tumorigenic. (a) Light image microscopy for the morphology of mixed cell culture. Scale bar, $500 \mu \mathrm{m}$. (b) The mixed cell culture continued the normal proliferation kinetic. (c) FACS analysis showing the difference in marker expression between mixed cell culture (AD10Mix) and AD10. The high expression of CD56, SOX2, SOX9, and relative low expression of CD105 represents a marker panel that distinguishes mixed culture from regular non-tumorigenic serum culture. FACS gate was set on total population after excluding cell debris and doublets. (d) Hybrid FACS expression profile for the four mixed cell cultures derived from the four patient samples that generate spheres in sphere conditions. Error bar indicates mean \pm s.e.m. (e) Kaplan-Meier survival analysis of mixed cell culture compared with cells from different culture conditions with the representative sample T1341. T1341 AD10Mix (red), Sp (blue), AD1(purple) and AD10 (green). The mixed cell culture took a longer time than Sp to develop a tumor $(P<0.01)$. (f) A fluorescent overview image and zoom-in field of a tumor induced by mixed cell culture stained with human-specific nestin (red) and DAPI showing a large tumor that infiltrated the second hemisphere. Scale bar, $500 \mu \mathrm{m}$. (g) A fluorescent overview image and zoom-in field of tumor induced by mixed cell culture stained with CD31 (red), CD248 (green) and $\alpha$ SMA (purple) showing the angiogenesis. Scale bar, $500 \mu$ M. 

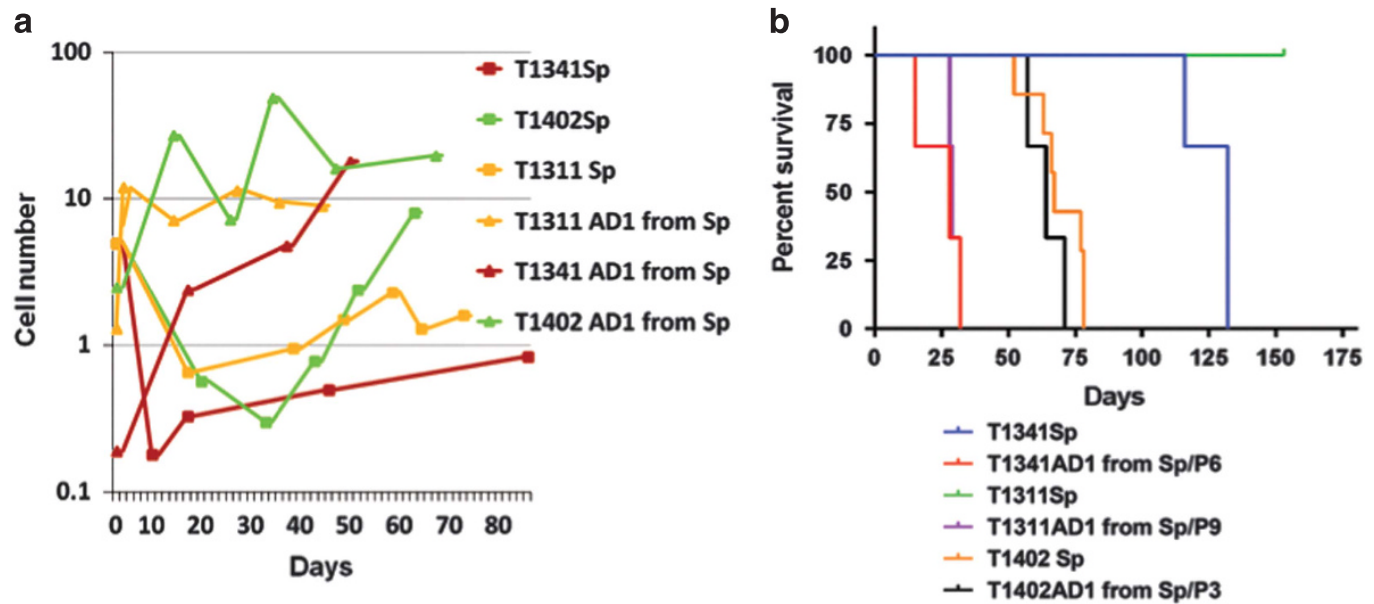

C
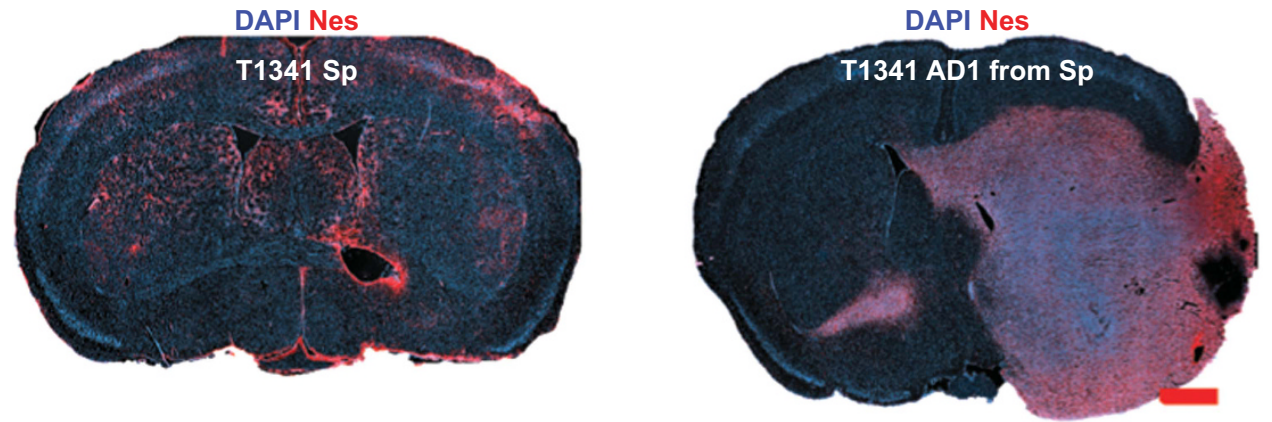

d

DAPI CD248 CD31 aSMA
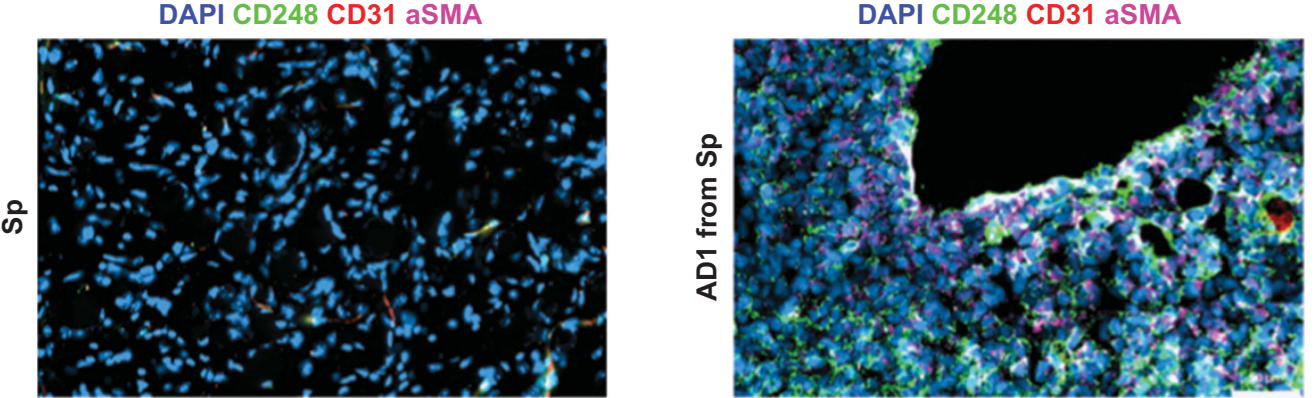

e

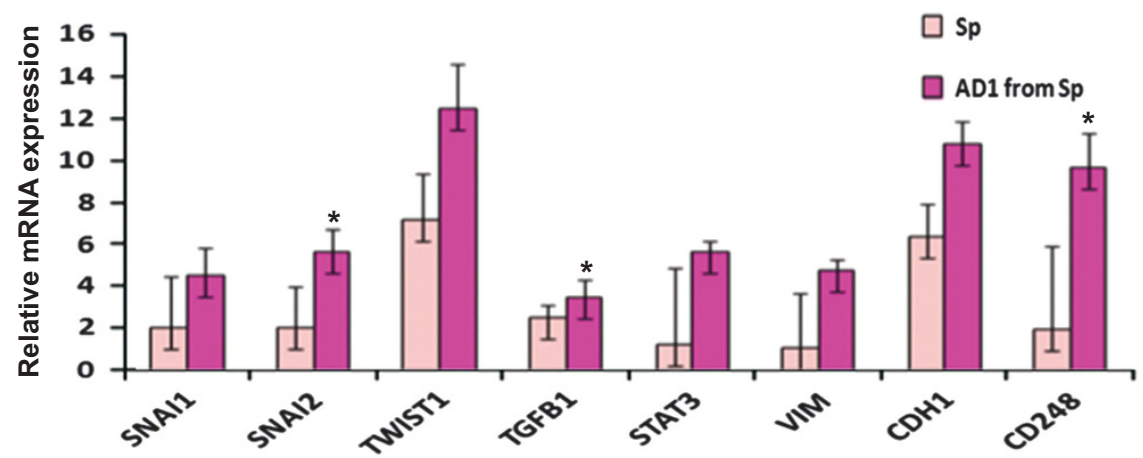

Figure 7. Moving cells established in sphere condition into AD1 accelerated the cell proliferation kinetics in vitro and in vivo and induced more angiogenic tumor. (a) Cell proliferation kinetics of Sp cells derived from three different patients after being moved to AD1 condition (AD1 from Sp). The cells acquired a higher proliferation rate in the new condition. (b) Kaplan-Meier survival analysis of Sp and AD1 from Sp. Cells from three different patients in both conditions were injected intracranially (100 000 cells in $4 \mu$ l per mouse), three to four mice in each group. T1341 and T1311 were kept longer in AD1 condition and transplanted after P6 or P9, respectively. The tumors formed by these cultures took a significantly shorter time than the original Sp $(P<0.003$ for T1341 and $P<0.0002$ for T1311). (c) An overview image of tumors induced by Sp and AD1 from Sp. Tissue sections are stained with human-specific nestin (red) and DAPI. Scale bar, $500 \mu \mathrm{m}$. (d) Immunofluorescent staining of CD31(red), CD248 (green), $\alpha$ SMA (purple) and DAPI of Sp and AD1 from Sp. The tumor induced by AD1 from Sp is more angiogenic. Scale bar, $50 \mu \mathrm{m}$. (e) Quantitative reverse transcription-PCR anlaysis of EMT-related genes and CD248 show that moving Sp cells into AD1 conditions results in relative increased expression of EMT genes, these were done on three different patients. The value is mean \pm s.e.m. 
Thus, the idea of having one universal marker for GSCs is a big challenge. Our results show that the tumor-forming cells express high levels of CD56, SOX2 and SOX9, while CD15 and CD133 are specific, but absent in a high proportion of samples. On the other hand, the non-tumorigenic stromal cells express high level of CD105, CD248 and aSMA. Therefore, we suggest that a panel set of CD56, SOX2, SOX9, CD105, CD248 and aSMA could cover the heterogeneity among GBM patients.

\section{Mesenchymal subtype of GSCs growing adherently}

One third of the samples in this study grew adherently under sphere condition. This growth pattern was reported previously. ${ }^{20,27}$ The difference in growth pattern between cells in sphere conditions most likely reflects differences in adhesion properties. Lottaz et al. $^{36}$ reported subtyping of $17 \mathrm{GSCs}$ into 2 groups: group I of GSCs was $\mathrm{CD} 133^{+}$and expressed the Proneural signature, while group II, grew semi-adherently, was CD133- and expressed a Mesenchymal signature. However, our data indicate that CD133 is not a perfect predictive marker for subtyping of GSCs. Rather, adherent growth in sphere conditions was highly specific for cells of the Mesenchymal subtype. Interestingly, our MRI analysis of the seven patient tumors that gave rise to the Sp-Mes subtype had the same pattern of invasion, characterized by contact with SVZ and spread into the white matter, without invasion into the cortex. ${ }^{22}$ Thus, the samples that grow adherently in sphere condition have a specific growth pattern in patients and in vitro, and express a mesenchymal signature. In our set of 118 DEGs between Sp and Sp-Mes, only 12 genes were shared with the TCGA 840-gene signature. ${ }^{26}$ Interestingly, 5 of these 12 genes were not in Bhat et al.'s $\mathrm{s}^{25}$ set of 1000 genes. These 12 genes alone separated the TCGA-GBM tissues into three groups: Mesenchymal identified by six genes, Classical by two genes and Proneural by four genes, whilst the TCGA Neural subtype predominantly expressed genes from both the Classical and Proneural subtypes. We propose that the 12-gene set can be used for simplified and quick subtyping of GBM tissues.

Primary mixed cell cultures in serum-containing medium are tumorigenic

Current dogma says that primary GBM cells cultured in serum conditions will not give rise to transplantable tumor-forming cells. ${ }^{11,37}$ Only when cells were cultivated up to high passage (>P10) and underwent in vitro transformation did they acquire tumorigenic potential with a different pathohistology from GBM. ${ }^{11}$ Exceptions are known for one case of a primary gliosarcoma cell culture, and for commercial GBM cell lines. ${ }^{15,16}$

Here, for the first time, we are introducing the concept of mixed cell culture. This term was chosen because non-adherent cells were allowed to remain in serum-containing cultures until the first medium change. This allowed adherent, mesenchymal cells to act as a feeder layer for the non-adherent, tumorigenic cells. This culture system worked well using both serum conditions, AD1 and AD10, and the cells were shown to be tumorigenic in P2-P9 of the tested cultures. It was previously suggested that dedifferentiation from a non-tumorigenic to a tumorigenic phenotype could explain tumorigenicity in a single case of gliosarcoma cells growing in serum conditions. ${ }^{15}$ Rather than dedifferentiation, we believe the explanation to be a cell culture strategy that allows the tumorigenic cells to remain in the culture. Some of our mixed cultures were kept for 6 months continuously in AD10 and still they had a high percentage of CD56-, SOX2-, SOX9-positive cells, and they were able to form spheres again. Using fresh tumor samples from the operation theatre directly without cryopreservation could be another reason for the retention of tumorigenicity in mixed cell cultures in serum. Finally, it is possible that secretion of GREM1 by the stromal cell feeder layer promotes tumor cell survival and proliferation in this system. ${ }^{38}$ We believe that the tumor cells derived from these cultures are true GBM cells and not in vitro-transformed cells because (1) the CGH analyses show imbalances also found in the parental fresh sample, (2) cells transplanted from early passage cultures gave rise to invasive tumors, and (3) the histology analyses showed invasive tumors crossing into the opposite hemisphere. Tumors derived from mixed cultures containing necrotic regions were highly angiogenic and enriched with pericytes.

In summary, we show that GBM cells enriched under classical serum cultures after removing non-adherent cells on day 2-3 are non-tumorigenic tumor stroma cells that lack the expression of GSCs markers. In contrast to this, a mixed cell culture in serum condition can be established by not removing floating cells. These cells express hybrid phenotype of GSC and MSC markers and are tumorigenic. Also, when cells growing as spheres are transferred to serum condition, $A D 1$, they retain their tumorigenic properties. Thus, the presence of serum does not impact on the tumorigenicity of GBM cells, as long as the cell culture strategy allows the tumorigenic cells to remain in the culture. Furthermore, we identify a marker panel that distinguishes tumorigenic from nontumorigenic cultures with: high SOX2, SOX9 and CD56, and low CD105, CD248 and aSMA. We also show that GBM cells that grow adherently in sphere condition (Sp-Mes) have mesenchymal properties. Comparing Sp-Mes cultures with sphere cultures of the Proneural subtype allowed us to identify 12 genes that alone were sufficient to subtype the TCGA-GBM tissue samples.

\section{MATERIALS AND METHODS}

Tumor patient sampling and cell culture

The brain tumor samples were acquired from 31 consenting GBM patients, human GBM. Only 21 patient samples were able to be grown in sphere conditions and these were used in this study. All experimental procedures were carried out according to the guide of the Norwegian National Committee for Medical Research Ethics after being approved by the Regional Ethical Committee (REC South-East S-07321d). Tumors were histologically classified as GBM according to World Health Organization (WHO) criteria. The cells were obtained by ultrasonic aspiration with a Sonoca 300 ultrasonic dissector/aspirator (Söring, Quickborn, Germany) perioperatively. Cells were washed and spun down to remove erythrocytes. Tumor cells were grown in sphere condition (serum free) containing DMEM/F12-GlutaMAX medium (Invitrogen, Paisley, UK), basic fibroblast growth factor $10 \mathrm{ng} / \mathrm{ml}$, epidermal growth factor $20 \mathrm{ng} / \mathrm{ml}$ (both R\&D Systems, Minneapolis, MN, USA), B27-supplement 1:50 (Invitrogen), Penicillin/Streptomycin $100 \mathrm{U} / \mathrm{ml}$ of both (Lonza, Basel, Switzerland), Heparin $1 \mathrm{ng} / \mathrm{ml}$ (Leo Pharma, Ballerup, Denmark) and HEPES 5 mm (Lonza, Vervier, Belgium), and in two serum conditions: (1) DMEM/F12-GlutaMAX medium supplemented with $1 \% \mathrm{FBS}, 2 \% \mathrm{~B}-27,10 \mathrm{ng} / \mathrm{ml}$ basic fibroblast growth factor, $20 \mathrm{ng} / \mathrm{ml}$ TGFa, $10 \mathrm{~mm}$ HEPES (Lonza, BioWhittaker) and $2.5 \mu \mathrm{g} / \mathrm{ml}$ heparin (Leo Pharma AS, Esbjerg, Denmark); ${ }^{21}$ (2) DMEM/F12GlutaMAX medium, $10 \%$ FBS. The cells were seeded at a density of 13000 cells $/ \mathrm{cm}^{2}$. In regular cell culture practice, we change the medium $48-72 \mathrm{~h}$ after plating the cells from fresh tumor samples and wash twice with PBS to clean away dead cells and cell debris, so all non-adherent cells are thrown away. For mixed culture, we did not remove non-adherent cells, and the medium was changed gently without washing.

\section{MR image analysis}

T1-wieghted images with contrast enhancement, and T2-weighted images were obtained and analyzed for each of the glioblastoma tumors. In addition, diffusion-weighted imaging together with apparent diffusion coefficient images was analyzed as well for the same GBM species.

\section{Flow cytometry analysis}

Cells from fresh samples that were processed into single cells without being in culture and cultured cells from different conditions were first washed with FACS buffer (PBS containing 4\% FBS). (Supplementary Method and Supplementary Method Tables) showing the antibodies that were used for surface and intracellular staining. For intracellular staining, cells were first fixed and permeabilized using BD Cytofix/Cytoperm Kit 
(Torreyana Rd, San Diego, CA, USA), and then incubated overnight with primary antibodies, after washing cells were incubated with matched secondary antibodies for $2 \mathrm{~h}$. Cells were then washed before being analyzed by flow cytometer, LSRFortessa cell analyzer (BD Bioscience, San Jose, CA, USA). At least 10000 events were counted.
In vitro characterization of GSCs and MSCs

Single-cell self-renewal. Cells from sphere cultures and serum cultures were used for sorting. Cells were collected by trypsin-EDTA $(0.25 \%)$ treatment, washed, centrifuged and resuspended in FACS buffer at a concentration of $1 \times 10^{6}$ cells per $500 \mu$ l. Single cells were sorted by

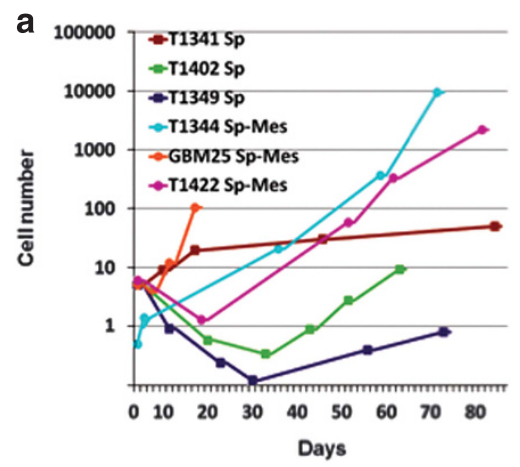

C

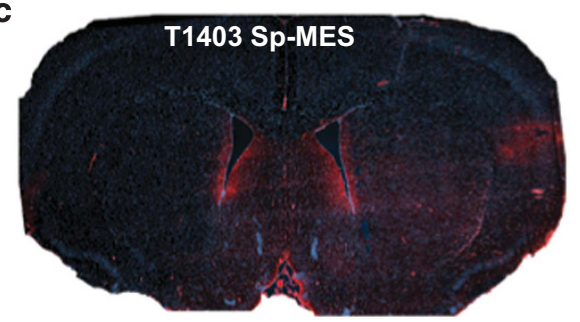

b
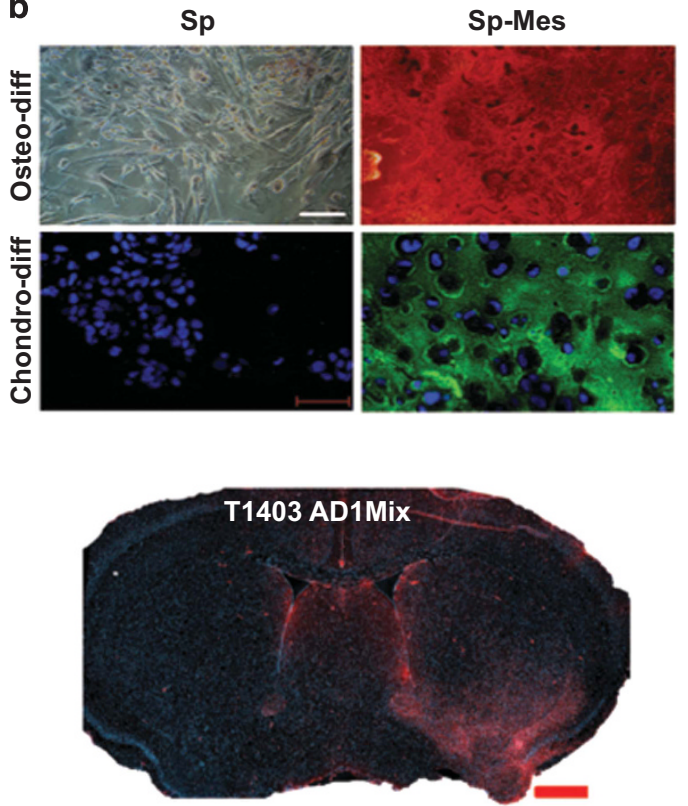

d

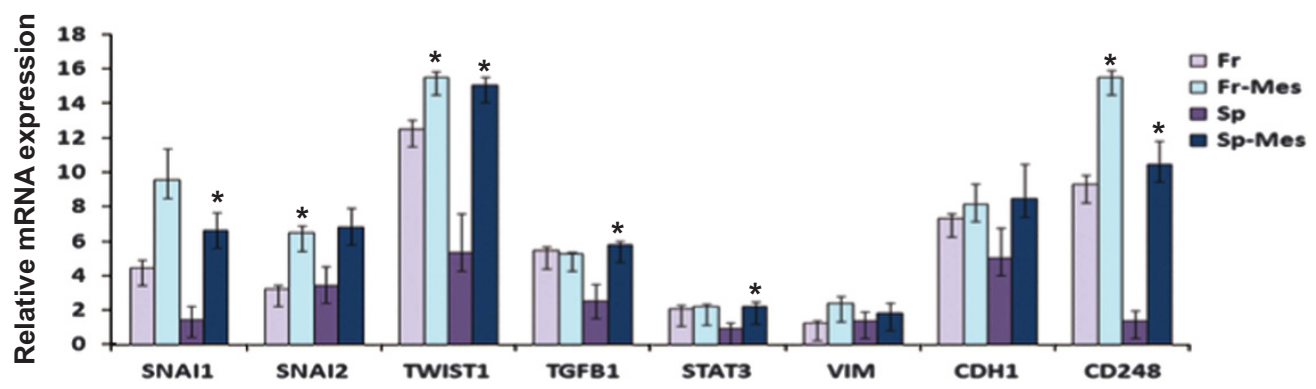

e

\section{f}
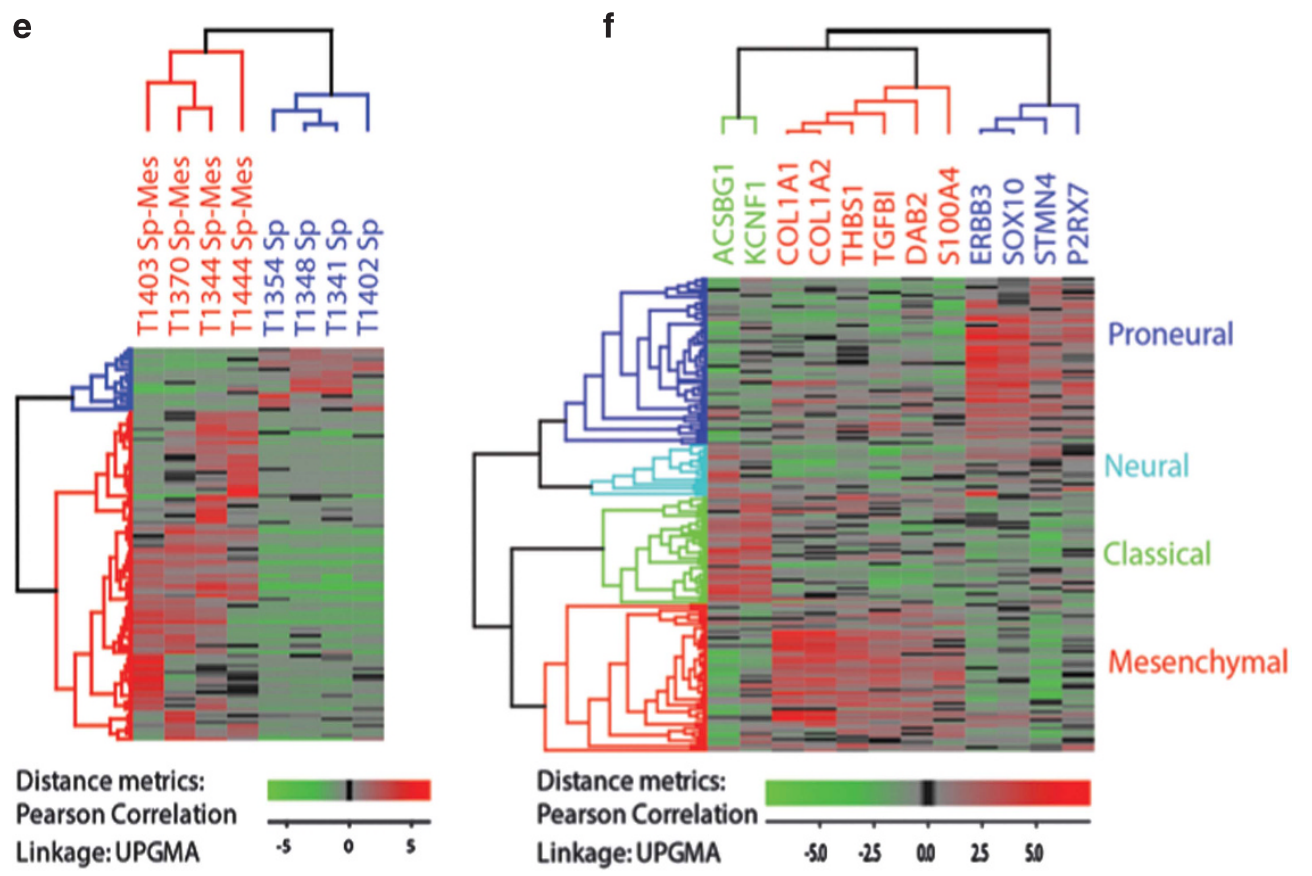

Distance metrics: Pearson Correlation Linkage:UPGMA

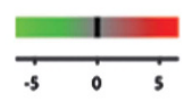


Figure 8. Sphere cultures that grow adherently (Sp-Mes) express mesenchymal properties and belong to mesenchymal subtype GSCs. The 12gene signature can subtype the TCGA samples in a manner similar to the 840-gene identifier TCGA-gene set. (a) Cell proliferation kinetics of $\mathrm{Sp}$-Mes compared with Sp cultures group, three cultures from different patients are represented for each group. (b) Bilineage mesenchymal differentiation of Sp and Sp-Mes into osteoblasts and chondrocytes. The Sp-Mes showed strong differentiation capacity into osteoblast (first row) and chondrocytes (second row), contrary to Sp cultures. Osteo-differentiation is visualized with red staining for calcium deposits. Scale bar, $250 \mu \mathrm{m}$. Chondrocytes are stained for aggrecan. Scale bar, $50 \mu \mathrm{m}$. (c) A fluorescent overview image of tumors induced from Sp-Mes and a mixed culture of this tumor AD1Mix. Tissue sections are stained with human-specific nestin (red) and DAPI. Scale bar, $500 \mu \mathrm{m}$. (d) Relative mRNA expression by quantitative reverse transcription-PCR for EMT-related genes and CD248 as a pericyte marker show that Sp-Mes and the original fresh tumor (Fr-Mes) have higher EMT profile. A significant difference was seen between cultured Sp and Sp-Mes in most genes SNAl1, SNAI2, TWIST1, TGF- $\beta$ and STAT3, while CDH1 tends to be higher in Sp-Mes, but not significantly. CD248 was significantly higher in Fr-Mes and corresponding Sp-Mes. The value is mean \pm s.e.m. Mann-Whitney test was used for statistical significance $\left({ }^{*} P<0.05\right)$. (e) Comparing transcriptional profiles of the Sp group with those of the Sp-Mes group identified 118 genes that were differentially expressed genes (DEGs). Supervised hierarchical clustering using this set confirmed separation into two groups. Red and green colors indicate highly and lowly expressed genes, respectively. (f) Hierarchical clustering using our 12-gene signature resembles the TCGA classification. The 12-gene signature was created by merging our set of 118 DEGs with the TCGA identifier set (840 genes). The 12 genes present in both sets could classify GBM tissues of TCGA data set into three subtypes: proneural identified with four genes (P2RX7, STMN4, SOX10 and ERBB3), classical with two genes (ACSBG1 and KCNF1) and mesenchymal with six genes (S100A, DAB2, TGFB1, THBS1, COL1A2 and COL1A1). The GBM samples of neural subtype were fused with proneural and classical subtypes mainly and did not have specific genes in our 12-gene mini signature.

FACS into a 96-well plate (1-3 cells/well) and were grown in sphere conditions or serum conditions for 3 weeks to generate the first generation, then the resultant sphere or colonies were trypsinized and plated using a limited dilution assay, to get second generation and then third generation cells.

Neural differentiation. Single cells dissociated from GSC- and MSC-like cells were plated in DMEM/F12-GlutaMAX medium (Invitrogene, Paisley, Scotland, UK) supplemented with $4 \%$ FBS, pencillin/streptomycin, HEPES, B27-supplemented with retinoic acid.

Osteoblastic differentiation. Cells were plated in triplicate at 10000 cells/well in 24-well plates. Cultured cells were grown in osteogenic differentiation medium (Grand Island, NY, USA) for 2 weeks. Undifferentiated cells in original growth medium were used as a negative control, BM- and AT-MSCs were used as positive controls. Alizarin red staining was used to visualize calcium deposition.

Adipogenic differentiation. Cells were plated in triplicate at $20000 /$ well in 24-well plates. Cultured Cells were grown in adipogenic differentiation medium as previously reported..$^{10}$ Undifferentiated cells in original growth medium were used as negative control, and BM- and AT-MSCs were used as positive controls. ${ }^{28}$ The induction of adipocytes was assessed after 2 weeks using oil red O stain (Sigma-Aldrich, Co, St Louis, MO, USA) as an indicator of intracellular lipid accumulation.

Chondrogenic differentiation. Chondrogenic induction was performed by pelleting cultured cells $\left(5 \times 10^{5}\right.$ cells per pellet) and then by putting them through the process of preparing alginate scaffold following an established protocol. ${ }^{28}$ Chondrocyte differentiation was evaluated by aggrecan and Collagen-2 staining after 3 weeks of differentiation.

In all three assays, the medium was replaced twice a week and controls were grown in the original medium for each condition. ${ }^{10}$

\section{In vivo transplantation}

The experimental protocol was approved by the Norwegian National Animal Research Authority project licence no FOTS-id 4785 and 5940. The animal experiments were performed in accordance with the European Convention for the Protection of Vertebrate Animals used for Experimental and Other Scientific Purposes (ETS 123) and The Guide for the Care and Use of Laboratory Animals. ${ }^{39}$ Six to 8-week-old SCID mice were purchased from Scanbur (Scanbur AS, Karlslunde, Denmark). About 100000 dissociated tumor cells in $4 \mu$ l neurobasal medium were intracranially injected in the right striatum of SCID mice $(0.5 \mathrm{~mm}$ forward from bregma, $1.5 \mathrm{~mm}$ lateral and $2.5 \mathrm{~mm}$ ventral from the dura) using a $10 \mu \mathrm{l}$ Hamilton syringe with a 33-gauge needle. To inhibit the back-flow through the injection canal, the needle was left in the brain for $2 \mathrm{~min}$ and withdrawn gradually $(1 \mathrm{~mm}$ with waiting time for $2 \mathrm{~min}$ ). Mice were allowed to recover; their health status was closely monitored, and immediately killed when they started showing neurological symptoms.

\section{Statistical and bioinformatic analysis}

Survival analysis and the Mantel-Cox log-rank were performed using GraphPad Prism 6 (GraphPad Software, LA Jolla, CA, USA). The quantification in the marker panel is shown as a percentage of the mean \pm s.e.m. Quantitative PCR data were analyzed using the Mann-Whitney test, and a $P$-value $<0.05$ was considered significant.

\section{CONFLICT OF INTEREST}

The authors declare no conflict of interest.

\section{ACKNOWLEDGEMENTS}

We thank Neurosurgery Department and Institute for Surgical Research for provision of patients' samples and financial support. Grazyna Babinska, Hong Qu, Birthe Mikkelsen, Aphirak Juthajan and Petter Brandal for technical support; Yan Zhang for flow cytometry core facility. High-resolution histological images were acquired at the Norbrain Slide Scanning Facility at the Institute of Basic Medical Sciences, the University of Oslo, funded by the Research Council of Norway. We also thank Marianne Hanna for participating in the graphic drawing; Wayne Murrell for improving the language clarity; Jan Helge Solbakk and Peter Kierulf for guidance. This work was funded by Helse Sør-Øst and Oslo University Hospital. Microarray data have been deposited in the Gene Expression Omnibus (GEO) database (accession number GSE74571).

\section{AUTHOR CONTRIBUTIONS}

JB was involved in conception and design, conducting experiments, data collection and assembly, data analysis and interpretation and manuscript writing; BS was involved in microarray data analysis and interpretation, and manuscript writing; SAMH was involved in data collection and MRI analysis; MJ was involved in conducting experiment and manuscript writing; TKO and FM was involved in CGH analysis; JCG was involved in data interpretation and manuscript writing; PI was involved in conducting experiments, data analysis and interpretation and manuscript writing; JB was involved in conception and design, data analysis and interpretation and manuscript writing.

\section{REFERENCES}

1 Louis DN, Ohgaki H, Wiestler OD, Cavenee WK, Burger PC, Jouvet A et al. The 2007 WHO classification of tumours of the central nervous system. Acta Neuropathol 2007; 114: 97-109.

2 Bao S, Wu Q, McLendon RE, Hao Y, Shi Q, Hjelmeland AB et al. Glioma stem cells promote radioresistance by preferential activation of the DNA damage response. Nature 2006; 444: 756-760.

3 Hadjipanayis CG, Van Meir EG. Tumor initiating cells in malignant gliomas: biology and implications for therapy. J Mol Med (Berl) 2009; 87: 363-374.

4 Du R, Lu KV, Petritsch C, Liu P, Ganss R, Passegué E et al. HIF1alpha induces the recruitment of bone marrow-derived vascular modulatory cells to regulate tumor angiogenesis and invasion. Cancer Cell 2008; 13: 206-220. 
5 Junttila MR, de Sauvage FJ. Influence of tumour micro-environment heterogeneity on therapeutic response. Nature 2013; 501: 346-354.

6 Charles NA, Holland EC, Gilbertson R, Glass R, Kettenmann H. The brain tumor microenvironment. Glia 2012; 60: 502-514.

7 Ricci-Vitiani L, Pallini R, Biffoni M, Todaro M, Invernici G, Cenci T et al. Tumour vascularization via endothelial differentiation of glioblastoma stem-like cells. Nature 2010; 468: 824-828.

8 Cheng L, Huang Z, Zhou W, Wu Q, Donnola S, Liu JK et al. Glioblastoma stem cells generate vascular pericytes to support vessel function and tumor growth. Cell 2013; 153: 139-152.

9 Pyonteck SM, Akkari L, Schuhmacher AJ, Bowman RL, Sevenich L, Quail DF et al. CSF-1 R inhibition alters macrophage polarization and blocks glioma progression. Nat Med 2013; 19: 1264-1272.

10 Behnan J, Isakson P, Joel M, Cilio C, Langmoen IA, Vik-Mo EO et al. Recruited brain tumor-derived mesenchymal stem cells contribute to brain tumor progression. Stem Cells 2014; 32: 1110-1123.

11 Lee J, Kotliarova S, Kotliarov Y, Li A, Su Q, Donin NM et al. Tumor stem cells derived from glioblastomas cultured in bFGF and EGF more closely mirror the phenotype and genotype of primary tumors than do serum-cultured cell lines. Cancer Cell 2006; 9: 391-403.

12 Pollard SM, Yoshikawa K, Clarke ID, Danovi D, Stricker S, Russell R et al. Glioma stem cell lines expanded in adherent culture have tumor-specific phenotypes and are suitable for chemical and genetic screens. Cell Stem Cell 2009; 4: 568-580.

13 Galli R, Binda E, Orfanelli U, Cipelletti B, Gritti A, De Vitis S et al. Isolation and characterization of tumorigenic, stem-like neural precursors from human glioblastoma. Cancer Res 2004; 64: 7011-7021.

14 Patel AP, Tirosh I, Trombetta JJ, Shalek AK, Gillespie SM, Wakimoto $\mathrm{H}$ et al. Single-cell RNA-seq highlights intratumoral heterogeneity in primary glioblastoma. Science 2014; 344: 1396-1401.

15 deCarvalho AC, Nelson K, Lemke N, Lehman NL, Arbab AS, Kalkanis S et al. Gliosarcoma stem cells undergo glial and mesenchymal differentiation in vivo. Stem Cells 2010; 28: 181-190.

16 Hong X, Chedid K, Kalkanis SN. Glioblastoma cell line-derived spheres in serumcontaining medium versus serum-free medium: a comparison of cancer stem cell properties. Int J Oncol 2012; 41: 1693-1700.

17 Stoczynska-Fidelus $E$, Bienkowski M, Pacholczyk M, Winiecka-Klimek $M$, Banaszczyk M, Zieba J et al. Different mutational characteristics of TSG in cell lines and surgical specimens. Tumour Biol 2014; 35: 11311-11318.

18 Singh SK, Hawkins C, Clarke ID, Squire JA, Bayani J, Hide T et al. Identification of human brain tumour initiating cells. Nature 2004; 432: 396-401.

19 Ogden AT, Waziri AE, Lochhead RA, Fusco D, Lopez K, Ellis JA et al. Identification of A2B5+CD133- tumor-initiating cells in adult human gliomas. Neurosurgery 2008; 62: 505-514; discussion 514-505.

20 Beier D, Hau P, Proescholdt M, Lohmeier A, Wischhusen J, Oefner PJ et al. CD133 (+) and CD133(-) glioblastoma-derived cancer stem cells show differential growth characteristics and molecular profiles. Cancer Res 2007; 67: 4010-4015.

21 Murrell W, Palmero E, Bianco J, Stangeland B, Joel M, Paulson L et al. Expansion of multipotent stem cells from the adult human brain. PLoS One 2013; 8: e71334.

22 Lim DA, Cha S, Mayo MC, Chen MH, Keles E, VandenBerg S et al. Relationship of glioblastoma multiforme to neural stem cell regions predicts invasive and multifocal tumor phenotype. Neuro Oncol 2007; 9: 424-429.

23 Worthley DL, Churchill M, Compton JT, Tailor Y, Rao M, Si Y et al. Gremlin 1 identifies a skeletal stem cell with bone, cartilage, and reticular stromal potential. Cell 2015; 160: 269-284.

24 Dumont N, Wilson MB, Crawford YG, Reynolds PA, Sigaroudinia M, Tlsty TD. Sustained induction of epithelial to mesenchymal transition activates DNA methylation of genes silenced in basal-like breast cancers. Proc Natl Acad Sci USA 2008; 105: 14867-14872.
25 Bhat KP, Balasubramaniyan V, Vaillant B, Ezhilarasan R, Hummelink K, Hollingsworth $\mathrm{F}$ et al. Mesenchymal differentiation mediated by NF-kappaB promotes radiation resistance in glioblastoma. Cancer Cell 2013; 24: 331-346.

26 Verhaak RG, Hoadley KA, Purdom E, Wang V, Qi Y, Wilkerson MD et al. Integrated genomic analysis identifies clinically relevant subtypes of glioblastoma characterized by abnormalities in PDGFRA, IDH1, EGFR, and NF1. Cancer Cell 2010; 17: 98-110.

27 Gunther HS, Schmidt NO, Phillips HS, Kemming D, Kharbanda S, Soriano R et al. Glioblastoma-derived stem cell-enriched cultures form distinct subgroups according to molecular and phenotypic criteria. Oncogene 2008; 27: 2897-2909.

28 Herlofsen SR, Kuchler AM, Melvik JE, Brinchmann JE. Chondrogenic differentiation of human bone marrow-derived mesenchymal stem cells in self-gelling alginate discs reveals novel chondrogenic signature gene clusters. Tissue Eng Part A 2011; 17: 1003-1013.

29 Soeda A, Hara A, Kunisada T, Yoshimura S, Iwama T, Park DM. The evidence of glioblastoma heterogeneity. Sci Rep 2015; 5: 7979.

30 Hagerstrand D, He X, Bradic Lindh M, Hoefs S, Hesselager G, Ostman A et al. Identification of a SOX2-dependent subset of tumor- and sphere-forming glioblastoma cells with a distinct tyrosine kinase inhibitor sensitivity profile. Neuro Oncol 2011; 13: 1178-1191.

31 Wang L, He S, Yuan J, Mao X, Cao Y, Zong J et al. Oncogenic role of SOX9 expression in human malignant glioma. Med Oncol 2012; 29: 3484-3490.

32 Clara CA, Marie SK, de Almeida JR, Wakamatsu A, Oba-Shinjo SM, Uno M et al. Angiogenesis and expression of PDGF-C, VEGF, CD105 and HIF-1alpha in human glioblastoma. Neuropathology 2014; 34: 343-352.

33 Kemper K, Sprick MR, de Bree M, Scopelliti A, Vermeulen L, Hoek M et al. The AC133 epitope, but not the CD133 protein, is lost upon cancer stem cell differentiation. Cancer Res 2010; 70: 719-729.

34 Barrantes-Freer A, Renovanz M, Eich M, Braukmann A, Sprang B, Spirin P et al. CD133 expression is not synonymous to immunoreactivity for $\mathrm{AC} 133$ and fluctuates throughout the cell cycle in glioma stem-like cells. PLoS One 2015; 10: e0130519.

35 Son MJ, Woolard K, Nam DH, Lee J, Fine HA. SSEA-1 is an enrichment marker for tumor-initiating cells in human glioblastoma. Cell Stem Cell 2009; 4: 440-452.

36 Lottaz C, Beier D, Meyer K, Kumar P, Hermann A, Schwarz J et al. Transcriptional profiles of CD133+ and CD133- glioblastoma-derived cancer stem cell lines suggest different cells of origin. Cancer Res 2010; 70: 2030-2040.

37 Hossain A, Gumin J, Gao F, Figueroa J, Shinojima N, Takezaki T et al. Mesenchymal stem cells isolated from human gliomas increase proliferation and maintain stemness of glioma stem cells through the IL-6/gp130/STAT3 Pathway. Stem Cells 2015; 33: 2400-2415

38 Sneddon JB, Zhen $\mathrm{HH}$, Montgomery $\mathrm{K}$, van de Rijn $\mathrm{M}$, Tward $\mathrm{AD}$, West $\mathrm{R}$ et al. Bone morphogenetic protein antagonist gremlin 1 is widely expressed by cancerassociated stromal cells and can promote tumor cell proliferation. Proc Natl Acad Sci USA 2006; 103: 14842-14847.

39 The Guide for the Care and Use of Laboratory Animals, 8th edn. The National Academic Press: Washington, DC, USA, NRC, 2011.

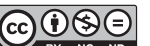

This work is licensed under a Creative Commons AttributionNonCommercial-NoDerivs 4.0 International License. The images or other third party material in this article are included in the article's Creative Commons license, unless indicated otherwise in the credit line; if the material is not included under the Creative Commons license, users will need to obtain permission from the license holder to reproduce the material. To view a copy of this license, visit http:// creativecommons.org/licenses/by-nc-nd/4.0/

(c) The Author(s) 2017

Supplementary Information accompanies this paper on the Oncogene website (http://www.nature.com/onc) 